\title{
Schwinger-Dyson equations in Coulomb gauge consistent with numerical simulation
}

\author{
Patrick Cooper $^{1, *}$ and Daniel Zwanziger ${ }^{2, \dagger}$ \\ ${ }^{1}$ Duquesne University, Pittsburgh, Pennsylvania 15282, USA \\ ${ }^{2}$ New York University, New York, New York 10003, USA
}

(Received 4 May 2018; published 10 December 2018)

\begin{abstract}
In the present work, we undertake a study of the Schwinger-Dyson equation (SDE) in the Euclidean formulation of local quantum gauge field theory, with Coulomb gauge condition $\partial_{i} A_{i}=0$. We continue a previous study which kept only instantaneous terms in the SDE that are proportional to $\delta(t)$ in order to calculate the instantaneous part of the time component of the gluon propagator $D_{A_{0} A_{0}}(t, R)$. We compare the results of that study with a numerical simulation of lattice gauge theory and find that the infrared critical exponents and related quantities agree to within $1 \%$ to $3 \%$. This raises the question, "Why is the agreement so good, despite the systematic neglect of noninstantaneous terms?" We discovered the happy circumstance that all the noninstantaneous terms are in fact zero. They are forbidden by the symmetry of the local action in Coulomb gauge under time-dependent gauge transformations $g(t)$. This remnant gauge symmetry is not fixed by the Coulomb gauge condition. The numerical result of the present calculation is the same as in the previous study; the novelty is that we now demonstrate that all the non-instantaneous terms in the SDE vanish. We derive some elementary properties of propagators which are a consequence of the remnant gauge symmetry. Our results support the simple physical scenario in which confinement is the result of a linearly rising color-Coulomb potential, $V(R) \sim \sigma R$ at large $R$. We also show that the horizon condition $\langle H(g A)\rangle=\left(N^{2}-1\right) d V$, and the divergence of the ghost dressing function at $\mathbf{k}=0, \lim _{|\mathbf{k} \rightarrow 0|} \mathbf{k}^{2} D_{c \bar{c}}(\mathbf{k})=\infty$, are identical gauge conditions.
\end{abstract}

DOI: 10.1103/PhysRevD.98.114006

\section{INTRODUCTION}

While the quest for exotic quantum theories of gravity captivates many physicists, a much more mundane question remains unanswered: what is the qualitative mechanism for the mismatch between the UV degrees of freedom (d.o.f.) of the standard model (quarks and gluons) and the IR states we observe in the lab (baryons and mesons). In other words, an intuitive physical picture of confinement still illudes us, despite the empirical successes of the standard model in the UV. Genuinely new physics is unlikely needed; from lattice simulations, we know that nonAbelian gauge theory by itself is capable of creating gluonic flux tubes which confine quark-antiquark pairs into mesons at low energy [1]. Yet despite our best efforts, the mathematics behind this phenomenon is unknown. The ultimate goal of science is not just to reproduce nature, but

\footnotetext{
cooperp@duq.edu

dz2@nyu.edu
}

Published by the American Physical Society under the terms of the Creative Commons Attribution 4.0 International license. Further distribution of this work must maintain attribution to the author(s) and the published article's title, journal citation, and DOI. Funded by SCOAP . rather to understand it, and this goal is what drives the field of nonperturbative QCD.

The breakdown of perturbation theory at low energies forces one to face the non-Abelian character of Yang-Mills theory head on. Various approaches have been made over the years to use functional methods to extract information about the fully nonperturbative, dressed propagators and vertices of QCD. These quantities are crucial to understanding confinement. For example, an infrared vanishing gluon propagator violates reflection positivity and, thus, implies that the gluon is not an asymptotic field of the theory. Also, in Landau gauge, the divergence of the ghost dressing function at $k=0$ leads to a well defined global color charge which is an important part of the Kugo-Ojima confinement scenario [2,3]. Two techniques that have greatly increased our understanding of the nonperturbative sector of QCD, constituting an infinite hierarchy of coupled equations that can be derived rigorously from the full quantum effective action, are the functional renormalization group equations (FRG) $[2,4-6]$ and the SchwingerDyson equations (SDE) [7-14]. A third technique, exploits a formal similarity between vacuum expectation values in the Hamiltonian formalism and correlation functions in Euclidean quantum field theory. In this approach, an ansatz is made for the vacuum wave functional which confirms 
results found by other techniques [4,15-20]. The advantage of the canonical approach is that with Lagrangian methods, an uncontrolled truncation must be made to complete the equations. At first glance, it seems that in the Hamiltonian approach, a truncation is still made even with a nonGaussian ansatz: a finite order polynomial is still used for the vacuum wave functional. However, due to the gap equation found by varying the energy density, the best possible coefficients of that finite order polynomial will be found which minimizes the effect of the truncation [15]. Nonetheless, we will proceed with the approach povided by the Schwinger-Dyson equations. In contradistinction to the Hamiltonian operator method, we use a local Euclidean quantum field theory.

Coulomb gauge is a natural choice for attempting a qualitative understanding of confinement for two reasons. First, it is a unitary gauge, where Gauss's law can be resolved explicitly by the longitudinal component of the color electric field, thus only propagating physical d.o.f. (analogous to the two polarizations of the physical photon of QED). To interpolate between UV QCD and phenomenological theories of IR QCD, tracking the physical d.o.f. is essential. Second, the long-range nature of the color-Coulomb potential, $\delta\left(x_{0}-y_{0}\right) V_{\text {Coulomb }}(\vec{x}-\vec{y})=$ $\left\langle A_{0}(x) A_{0}(y)\right\rangle$, gives a physical picture of what does the confining. Despite being a gauge-dependent quantity, the color-Coulomb potential also gives us insight into the IR asymptotics of the gauge-invariant Wilson potential by the following argument, found in detail in [21]. Consider a quark-antiquark pair at separated points, $\vec{x}$ and $\vec{y}$ with $R \equiv|\vec{x}-\vec{y}|$. The correlator of two Wilson lines, $G(R, T)$, extending an amount $T$ in the time direction is related to the Hamiltonian and the state $\left|\psi_{\bar{q} q}\right\rangle$ by

$$
\begin{aligned}
G(R, T) & =\left\langle\frac{1}{2} \operatorname{Tr}\left[L^{\dagger}(\vec{x}, 0, T) L(\vec{y}, 0, T)\right]\right\rangle \\
& =\left\langle\psi_{\bar{q} q}\left|e^{-\left(H-E_{0}\right) T}\right| \psi_{\bar{q} q}\right\rangle,
\end{aligned}
$$

where $L(\vec{x}, 0, T)$ is a Wilson line extending from 0 to $T$ at point $\vec{x}$. Defining the logarithmic derivative,

$$
V(R, T)=-\frac{d}{d T} \log [G(R, T)],
$$

one can show that the Coulomb energy is obtained in the limit $T \rightarrow 0$, and the energy of the flux tube ground state is obtained in the opposite limit, $T \rightarrow \infty$. Since the latter is the ground state, at large $\mathrm{R}$ (so one can neglect the selfenergy contribution), $\quad V_{\text {Coulomb }}=V(R, 0)>V(R, \infty)=$ $V_{\text {Wilson }}$. Thus, the Coulomb potential must be at least linear (possibly super-linear) in order to reproduce a linearly rising Wilson potential like the one seen on the lattice. While a long range Coulomb potential is a necessary condition for confinement, it isn't a sufficient one.
Similarly to how charges screen each other to make neutral molecules despite the presence of the long range Coulomb potential, the QCD vacuum creates quark-antiquark pairs, confining color charge despite the presence of a long range color-Coulomb potential. Thus, even at high temperature, above the deconfinement phase transition, the long range Coulomb force is present as seen in [13].

The instantaneous character of the dynamics is of particular importance to those interested in studying the so-called quark-gluon plasma at high temperature. The common wisdom is that at high temperatures, typical momentum transfer is large, and thus, due to asymptotic freedom, quarks and gluons will behave like a weakly interacting plasma. The presence of a long range colorColoumb potential at high temperature challenges this view, and suggests that one might expect a strongly interacting fluid, despite the approximate StefanBoltzmann like behavior witnessed by Karsch et al. on the lattice [22]. This isn't contridictory with the renormalization group; recall that in Coulomb gauge, the physical quantity $g^{2} D_{A_{0} A_{0}}$ is a renormalization-group invariant [23]. This phenomenology would be similar to $\mathcal{N}=4$ super Yang-Mills (SYM) in the planar limit as pointed out in [24]. The comparison of high temperature QCD to $\mathcal{N}=4$ SYM, a strongly-coupled integrable theory, is particularly intriguing in light of an article by Dubovsky and Gorbenko [25] which suggests that at large $N$, the theory of QCD flux tubes may also be integrable, evading the no-go theorem in [26] by possessing a massless pseudoscalar mode in addition to the usual goldstone modes of a string-like flux tube embedded in spacetime. If the color-Coulomb potential is indeed stronger at high temperature than at zero temperature, as the lattice calculation suggests [21], this would imply that gluons are more likely to form color singlets (i.e., glueballs), rather than less, since gluon configurations not bound into their flux-tube ground state would be Boltzmann suppressed, making the flux tube description more relevant. The instantaneous character of the dynamics is crucial to accessing the physics at high temperature because it only keeps terms in correlation functions that dominate at vanishingly small temporal separation. At high temperatures, the partition function becomes vanishingly small in the Euclidean-time direction, thus yielding a dimensionally reduced theory, in addition to any instantaneous physics inherited from the higher dimensional theory. This heuristic picture is illustrated in [24] and a rigorous treatment of Gribov-Zwanger theory in Coulomb gauge at finite temperature can be found in [27].

One objective of this article is to gain a quantitative handle on the asymptotic behavior of the color-Coulomb potential. We do this by finding a self-consistent set of vertices of the full quantum effective action that satisfy the Schwinger-Dyson equations, continuing the work of [14]. More specifically, in that work, only terms in the SDEs were kept that are proportional to $\delta(t)$ in order to calculate 
the instantaneous part of $D_{A_{0} A_{0}}$, a.k.a., the color-Coulomb potential. In Sec. XI of the present article, we compare the infrared critical exponents found [14] with numerical simulation in lattice gauge theory of $S U(2)$ by Langfeld and Moyaerts [28]. The agreement is striking. There is also reasonably good agreement with Burgio, Quandt and Reinhardt [29] for $S U(2)$, and with Nakagawa et al. [30] for $S U(3)$. This led us to question why the agreement was so good, in view of the neglect of the noninstantaneous terms. We have discovered that the noninstantaneous terms vanish because of the invariance under time-dependent gauge transformations $g(t)$. These form the remnant gauge symmetry group of gauge transformations that are not fixed by the Coulomb gauge condition $\partial_{i} A_{i}=0$.

\section{LOCAL ON-SHELL FADDEEV-POPOV ACTION IN COULOMB GAUGE}

The Faddeev-Popov quantization of Yang-Mills theory in Coulomb gauge is defined in phase-space formalism by the Lagrangian density,

$$
\begin{aligned}
\mathcal{L}^{\mathrm{FP}}= & i \pi_{i}\left(D_{0} A_{i}-\partial_{i} A_{0}\right)+\frac{1}{2} \pi_{i}^{2}+(1 / 4) F_{i j}^{2} \\
& -\partial_{i} \bar{c} \cdot D_{i} c+i \partial_{i} b \cdot A_{i},
\end{aligned}
$$

where $F_{\mu \nu}=\partial_{\mu} A_{\nu}-\partial_{\nu} A_{\mu}+A_{\mu} \times A_{\nu}$ is the Yang-Mills field strength [23]. The connection $A_{\mu}^{a}$ as well as the Nakanishi-Lautrup and Faddev-Popov ghost fields $b^{a}, c^{a}$ and $\bar{c}^{a}$ are all fields in the adjoint representation of the global $S U(N)$ color group. Color components are represented by Latin superscripts. To streamline notation we adopt the convention that $X \cdot Y \equiv \sum_{a} X^{a} Y^{a}$ and $(X \times Y)^{a} \equiv \sum_{b c} g f^{a b c} X^{b} Y^{c}$, where $f^{a b c}$ are the $s u(N)$ structure constants and $g$ is the gauge coupling. In this notation, the gauge-covariant derivative in the adjoint representation is $D_{\mu} X=\partial_{\mu} X+A_{\mu} \times X$. If one integrates out the canonically conjugate color-electric field $\pi_{i}$, one gets the Coulomb-gauge Faddeev-Popov Lagrangian density in the second-order formalism,

$$
\begin{aligned}
\mathcal{L}^{\mathrm{FP}}= & \frac{1}{2}\left(D_{0} A_{i}-\partial_{i} A_{0}\right)^{2}+(1 / 4) F_{i j}^{2} \\
& -\partial_{i} \bar{c} \cdot D_{i} c+i \partial_{i} b \cdot A_{i},
\end{aligned}
$$

Next, we integrate out the $b$-field, so the gauge condition is satisfied on-shell, and $\mathbf{A}$ is purely transverse,

$$
\partial_{i} A_{i}=0 .
$$

We separate the transverse and longitudinal parts of $\pi$,

$$
\pi_{i}=\tau_{i}-\partial_{i} \lambda,
$$

where $\partial_{i} \tau_{i}=0$. The Faddeev-Popov action with the onshell gauge condition is given by

$$
\begin{aligned}
S= & \int d^{d+1} x\left[i \tau_{i} \cdot D_{0} A_{i}+\frac{1}{2} \tau^{2}+(1 / 4) F_{i j}^{2}+\frac{1}{2}\left(\partial_{i} \lambda\right)^{2}\right. \\
& \left.+i \partial_{i} \lambda \cdot D_{i} A_{0}-\partial_{i} \bar{c} \cdot D_{i} c\right]
\end{aligned}
$$

where we have used $\int d^{d+1} x \tau_{i} \cdot \partial_{i} A_{0}=\int d^{d+1} x \partial_{i} \lambda \cdot \partial_{0} A_{i}=0$. The time derivative appears only in the first term, $\tau_{i} \cdot D_{0} A_{i}=\tau_{i} \cdot \partial_{0} A_{i}+\tau_{i} \cdot g A_{0} \times A_{i}$.

\section{TIME-DEPENDENT GAUGE TRANSFORMATIONS AND THEIR CONSEQUENCE FOR PROPAGATORS}

The gauge condition $\partial_{i} A_{i}=0$ does not fix timedependent gauge transformations $g(t)$. Moreover, the action $S$ is invariant under such gauge transformations,

$$
S\left({ }^{g} \Phi_{\alpha},{ }^{g} A_{0}\right)=S\left(\Phi_{\alpha}, A_{0}\right)
$$

where the fields transform according to

$$
\begin{aligned}
\Phi_{\alpha}(t, \mathbf{x}) \rightarrow{ }^{g} \Phi_{\alpha}(t, \mathbf{x}) & =g^{-1}(t) \Phi_{\alpha}(t, \mathbf{x}) g(t) \\
A_{0}(t, \mathbf{x}) \rightarrow{ }^{g} A_{0}(t, \mathbf{x}) & =g^{-1}(t) A_{0}(t, \mathbf{x}) g(t)+g^{-1}(t) \partial_{0} g(t)
\end{aligned}
$$

and $\Phi_{\alpha}=t^{a} \Phi_{\alpha}^{a}$ and $A_{0}=t^{a} A_{0}^{a}$. The $t^{a}$ are a basis of the Lie algebra of the gauge structure group, $\left[t^{a}, t^{b}\right]=f^{a b c} t^{c}$, and $\Phi_{\alpha}^{a}=\left(A_{i}^{a}, \tau_{i}^{a}, \lambda^{a}, c^{a}, \bar{c}^{a}\right)$ represents all fundamental fields besides $A_{0}^{a}$. Under these transformations, $F_{\mu \nu}$ and $\pi$ transform gauge covariantly, ${ }^{g} F_{\mu \nu}=g^{-1} F_{\mu \nu} g$, and ${ }^{g} \pi_{i}=g^{-1} \pi_{i} g$. (In general, it will be understood that $g=g(t)$.) A symmetry of the action implies that expectation values are invariant under the same symmetry transformation,

$$
\left\langle O\left({ }^{g} \Phi_{\alpha},{ }^{g} A_{0}\right)\right\rangle=\left\langle O\left(\Phi_{\alpha}, A_{0}\right)\right\rangle
$$

This symmetry is generally ignored in analytic calculations, because it is broken in usual approximation schemes. For example, it is not a symmetry of the tree-level theory. ${ }^{1}$ However it is a powerful symmetry.

Statement:-Let $\phi_{1}^{a}(x)$ and $\phi_{2}^{a}(y)$ be two fields that transform covariantly under time-dependent gauge transformations. Then their propagator has a $\delta$-function singularity in time

\footnotetext{
${ }^{1}$ Indeed, the tree-level Lagrangian density in Coulomb gauge contains a time derivative in the term $\frac{1}{2}\left(\partial_{0} A_{i}\right)^{2}$, and only in this term. Under the infinitesimal time-dependent gauge transformation $\delta A_{i}^{a}=f^{a b c} A_{i}^{b} \times \omega^{c}(t)$, this term breaks the symmetry, $\delta \frac{1}{2}\left(\partial_{0} A_{i}\right)^{2}=f^{a b c} \partial_{0} A_{i}^{a} A_{i}^{b} \partial_{0} \omega^{c}(t) \neq 0$. There is no other term in the tree-level Lagrangian with a time derivative to cancel this.
} 


$$
\left\langle\phi_{1}^{a}(x) \phi_{2}^{b}(y)\right\rangle=\delta^{a b} U_{12}(\mathbf{x}-\mathbf{y}) \delta\left(x_{0}-y_{0}\right) .
$$

The proof is immediate. The infinitesimal form of the timedependent gauge transformation, Eq. (3.2), is

$$
\delta \phi_{i}(x)=\omega\left(x_{0}\right) \times \phi_{i}(x),
$$

where $i=1,2$, and invariance under infinitesimal timedependent gauge transformations, Eq. (3.4), reads

$$
\begin{aligned}
\left\langle\delta\left[\phi_{1}^{a}(x) \phi_{2}^{b}(y)\right]\right\rangle= & \left\langle\left[\omega\left(x_{0}\right) \times \phi_{1}(x)\right]^{a} \phi_{2}^{b}(y)\right. \\
& \left.+\phi_{1}^{a}(x)\left[\omega\left(y_{0}\right) \times \phi_{2}(y)\right]^{b}\right\rangle=0 .
\end{aligned}
$$

Global gauge invariance, that is, for $g=$ const, implies that $\left\langle\phi_{1}^{a}(x) \phi_{2}^{b}(x)\right\rangle=\delta^{a b} \mathcal{D}(x-y)$, and we have

$$
f^{a c b}\left[\omega^{c}\left(x_{0}\right)-\omega^{c}\left(y_{0}\right)\right] \mathcal{D}(x-y)=0 .
$$

This holds for all $\omega(t)$. The general solution to this condition, which is a well defined distribution, is Eq. (3.5), as asserted. The proof holds for other nontrivial representations such as the fundamental representation. It also extends immediately to the lattice. Propagators whose time-dependence is given by $\delta\left(x_{0}-y_{0}\right)$ will be called "instantaneous."

\section{PROPAGATORS IN COULOMB GAUGE}

The scalar fields $A_{0}$ and $\lambda$ and the ghost pair $c$ and $\bar{c}$ appear at most quadratically in the action, Eq. (2.1), with fixed $A_{i}$ and $\tau_{i}$. To calculate the propagators of these fields, one may integrate out the fields $A_{0}$ and $\lambda$ or $c$ and $\bar{c}$ by Gaussian integration, and one obtains the well-known formulas

$$
\begin{aligned}
\delta^{a b} D_{c \bar{c}}(x-y)= & \left\langle\left(M^{-1}\right)^{a b}(x)\right\rangle \delta\left(x_{0}-y_{0}\right) \\
\delta^{a b} i D_{A_{0} \lambda}(x-y)= & \left\langle\left(M^{-1}\right)^{a b}(x)\right\rangle \delta\left(x_{0}-y_{0}\right) \\
& +i\left\langle A_{\text {Ophys }}^{a}(x) \lambda_{\text {phys }}^{b}(y)\right\rangle \\
\delta^{a b} D_{A_{0} A_{0}}(x-y)= & \left\langle K^{a b}(\mathbf{x}, \mathbf{y})\right\rangle \delta\left(x_{0}-y_{0}\right) \\
& +\left\langle A_{\text {Ophys }}^{a}(x) A_{\text {ophys }}^{b}(y)\right\rangle \\
\delta^{a b} D_{\lambda \lambda}(x-y)= & \left\langle\lambda_{\text {phys }}^{a}(x) \lambda_{\text {phys }}^{b}(y)\right\rangle,
\end{aligned}
$$

where

$$
M(\mathbf{A})=-D_{i}(\mathbf{A}) \cdot \partial_{i}
$$

is the d-dimensional Faddeev-Popov operator that depends only on the transverse dynamical field $A_{i}, K$ is the operator with kernel

$$
K^{a b}\left(\mathbf{x}, \mathbf{y} ; y_{0}\right)=\left[M^{-1}\left(-\nabla^{2}\right) M^{-1}\right]^{a b}\left(\mathbf{x}, \mathbf{y} ; y_{0}\right),
$$

and

$$
\begin{aligned}
\lambda_{\text {phys }}^{a}(x) & \equiv \int d^{d} y\left(M^{-1}\right)^{a b}\left(\mathbf{x}, \mathbf{y} ; x_{0}\right) \rho^{b}\left(\mathbf{y}, x_{0}\right) \\
i A_{0 \text { phys }}(x) & \equiv-\int d^{d} y K^{a b}\left(\mathbf{x}, \mathbf{y} ; x_{0}\right) \rho^{b}\left(\mathbf{y}, x_{0}\right),
\end{aligned}
$$

are the potentials produced by the color charge density $\rho \equiv g \tau_{i} \times A_{i}$ of the dynamical gluons (and of quarks, if quarks are present). The Faddeev-Popov operator is hermitian, $-D_{i}(\mathbf{A}) \partial_{i}=-\partial_{i} D_{i}(\mathbf{A})$, because $A_{i}$ is transverse, $\partial_{i} A_{i}=0$.

\section{SCHWINGER-DYSON EQUATIONS}

We wish to explore the hypothesis that there exists an asymptotic infrared limit of the DSE which is dominated by loops containing an instantaneous propagator. Details of the derivation of the SD equations are given in [14]. (There is a slight change of notation. The substitutions from [14] to the present article are $\phi \rightarrow \lambda, \pi_{i}^{T} \rightarrow \tau_{i}, A_{i}^{T} \rightarrow A_{i}$.)

The time derivative appears in the action Eq. (2.5) only once, in the canonical term $i \tau \cdot \partial_{0} A_{i}$, so the fields $\tau_{i}$ and $A_{i}$ propagate in time, and there is no instantaneous term, with factor $\delta\left(x_{0}-y_{0}\right)$, in the propagators $D_{A_{i} A_{j}}, D_{\tau_{i} \tau_{j}}, D_{A_{i} \tau_{j}}$. The only propagators with the instantaneous factor $\delta\left(x_{0}-y_{0}\right)$ occur in the Eqs. (4.1) for the scalar propagators. In the DSE, there are some loops that contain at least one factor of $\delta\left(x_{0}-y_{0}\right)$, and some loops that contain none. The DSE holds separately for each of these sets, and we shall retain only those loops that contain at least one factor of $\delta\left(x_{0}-y_{0}\right)$. (It will turn out happily that this gives us a closed system of equations.) Because the fourier transform of an instantaneous propagator is independent of $k_{0}$,

$\int d^{d} \mathbf{x} d x_{0} \exp \left[-i\left(k_{0} x_{0}+\mathbf{k} \cdot \mathbf{x}\right)\right] D(\mathbf{x}) \delta\left(x_{0}\right)=\tilde{D}(\mathbf{k})$,

we obtain the instantaneous parts by making the substitutions

$$
\begin{aligned}
D_{A_{0} A_{0}}\left(|\mathbf{k}|, k_{0}\right) & \rightarrow D_{A_{0} A_{0}}(|\mathbf{k}|) \\
i D_{A_{0} \lambda}\left(|\mathbf{k}|, k_{0}\right) & \rightarrow i D_{A_{0} \lambda}(|\mathbf{k}|)=D_{c \bar{c}}(|\mathbf{k}|) \\
D_{\lambda \lambda}\left(|\mathbf{k}|, k_{0}\right) & \rightarrow 0 .
\end{aligned}
$$

The SDE is represented graphically in Fig. 1. However most terms vanish. We discard those, and keep the remaining terms. The tree-level terms are retained. The renormalization term (penguin diagram) is canceled by a mass counter-term. Consider the other one-loop graph which is the product of two propagators. The possibilities are: both propagators are instantaneous, or one is instantaneous and the other is not, or neither is. If they are both instantaneous, such as $\delta^{2}\left(x_{0}-y_{0}\right) D_{\lambda A_{0}}(\mathbf{x}-\mathbf{y}) D_{\lambda A_{0}}(\mathbf{x}-\mathbf{y})$, there is a terrible divergence, characteristic of the Coulomb gauge. Fortunately these terms cancel, as we shall see shortly. If one propagator is instantaneous, such as 


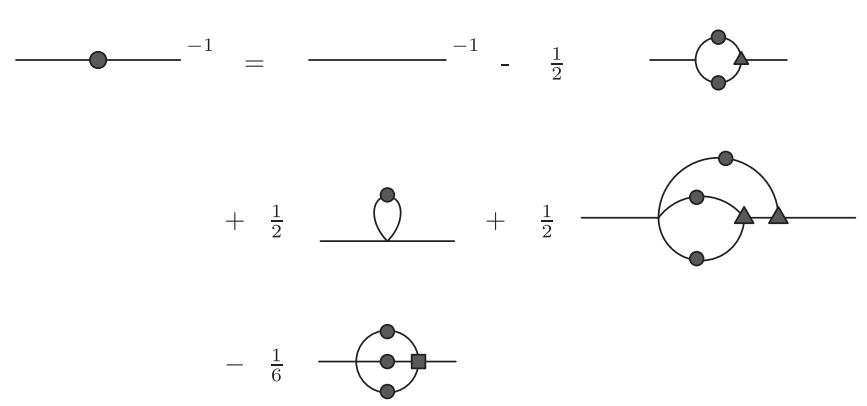

FIG. 1. Graphic representation of the SD equation. The undressed line and vertices represent tree-level quantities. The circles, triangles and square represent dressed propagators, dressed 3-vertices, and dressed 4-vertices respectively.

$V(\mathbf{x}-\mathbf{y}) \delta\left(x_{0}-y_{0}\right)$, and the other, $D_{N}(x-y)$, is noninstantaneous, the product is instantaneous,

$D_{N}(x-y) V(\mathbf{x}-\mathbf{y}) \delta\left(x_{0}-y_{0}\right)=U(\mathbf{x}-\mathbf{y}) \delta\left(x_{0}-y_{0}\right)$

where $U(\mathbf{x}-\mathbf{y})=D_{N}\left(\mathbf{x}-\mathbf{y}, x_{0}-y_{0}=0\right) V(\mathbf{x}-\mathbf{y})$, and gives an instantaneous contribution to $\Gamma$. The instantaneous one-loop graphs are represented in Fig. 2 (where the dressed 3 -vertices have been replaced by the tree-level 3-vertices, as will be discussed shortly). If both propagators in the loop are noninstantaneous, the result is neglected, because the product does not have a factor of $\delta\left(x_{0}-y_{0}\right)$, and is not instantaneous. Now consider the two two-loop graphs in Fig. 1. Both of these graphs contain a tree-level 4-vertex which originates from the quartic $\left(A_{i} \times A_{j}\right)^{2}$ term in the action Eq. (2.5). ${ }^{2}$ Three propagators emerge from the tree-level 4-vertex. Each of these propagators starts from the vector field $A_{i}$ so none of them is instantaneous. It follows that their product is not instantaneous, and their contribution may be neglected. So far our calculations are exact. We now make our only truncation: replace the remaining dressed 3-vertex (in the graph in Fig. 1) by the corresponding tree-level vertex. The result is given in Fig. 2 and in the following equations. This truncation has been explored in depth, and is found to be robust numerically in both Coulomb and Landau gauge [2,5-7,10,12,20,31-33]. In particular, for the ghost-gluon vertex in Coulomb gauge, this results from two properties [23]: (1) The external ghost momenta factor out of the corresponding Feynman integrals. This depresses the degree of convergence of the integrals, so (2) the vertex does not require renormalization $\tilde{Z}_{1}=1$. These properties severely restrict the allowed form of the complete vertex, and investigation did not reveal a new acceptable solution of the SD equation [14]. The same properties hold in the Landau gauge $[34,35]$. See the literature mentioned above for numerical support of this truncation for other vertices (e.g., the three gluon vertex is discussed at length in [5]).

\footnotetext{
${ }^{2}$ There is no $\left(A_{0} \times A_{i}\right)^{2}$ term in the action. It is replaced by a cubic term in $i \pi_{i} F_{0 i}$.
}

The resulting equations are represented graphically in Fig. 2, and, analytically by

$$
\begin{aligned}
\Gamma_{\mathbf{A A}}(k)= & \mathbf{k}^{2}+\frac{N g^{2}}{(2 \pi)^{d+1}} \int d^{d+1} p \\
& \times\left[\frac{d-2+(\hat{k} \cdot \hat{p})^{2}}{d-1} D_{\tau \tau}(p) D_{A_{0} A_{0}}(k-p)\right. \\
& +\frac{1-(\hat{k} \cdot \hat{p})^{2}}{d-1} \mathbf{p}^{2}\left(D_{A_{0} A_{0}}(p) D_{\lambda \lambda}(k-p)\right. \\
& \left.\left.+D_{A_{0} \lambda}(p) D_{A_{0} \lambda}(k-p)+D_{c \bar{c}}(p) D_{c \bar{c}}(k-p)\right)\right]
\end{aligned}
$$

$$
\begin{aligned}
\Gamma_{\tau \tau}(k)= & 1+\frac{N g^{2}}{(2 \pi)^{d+1}} \int d^{d+1} p \frac{d-2+(\hat{k} \cdot \hat{p})^{2}}{d-1} \\
& \times D_{\mathbf{A A}}(p) D_{A_{0} A_{0}}(k-p)
\end{aligned}
$$

$$
\begin{aligned}
\Gamma_{\tau \mathbf{A}}(k)= & -k_{0}+\frac{N g^{2}}{(2 \pi)^{d+1}} \int d^{d+1} p \frac{d-2+(\hat{k} \cdot \hat{p})^{2}}{d-1} \\
& \times D_{\mathbf{A} \tau}(p) D_{A_{0} A_{0}}(k-p)
\end{aligned}
$$

$$
\begin{aligned}
\Gamma_{A_{0} A_{0}}(k)= & \frac{N g^{2}}{(2 \pi)^{d+1}} \int d^{d+1} p \mathbf{k}^{2}\left[1-(\hat{k} \cdot \hat{p})^{2}\right] \\
& \times D_{\mathbf{A A}}(p) D_{\lambda \lambda}(p-k) \\
\Gamma_{\lambda \lambda}(k)= & \mathbf{k}^{2}+\frac{N g^{2} \mathbf{k}^{2}}{(2 \pi)^{d+1}} \int d^{d+1} p\left[1-(\hat{k} \cdot \hat{p})^{2}\right] \\
& \times D_{\mathbf{A A}}(p) D_{A_{0} A_{0}}(p-k)
\end{aligned}
$$

$$
\begin{aligned}
\Gamma_{\lambda A_{0}}(k)= & i \mathbf{k}^{2}+\frac{N g^{2} \mathbf{k}^{2}}{(2 \pi)^{d+1}} \int d^{d+1} p\left[1-(\hat{k} \cdot \hat{p})^{2}\right] \\
& \times D_{\mathbf{A A}}(p) D_{A_{0} \lambda}(p-k)
\end{aligned}
$$

$\Gamma_{\lambda A_{0}}(k)=i \Gamma_{\bar{c} c}(k)$,

where $D_{c \bar{c}}=\Gamma_{\bar{c} c}^{-1}$, and $\hat{k}$ and $\hat{p}$ are unit vectors. Note that the vector propagators are functions of $p$, and the scalar propagators are functions of $k-p$. The terms on the lefthand side $\Gamma_{\alpha \beta}$ are the two-point functions of the quantum effective action and are inverse to the propagators

$$
\Gamma_{\alpha \beta} D_{\beta \gamma}=\delta_{\alpha \gamma},
$$

where the indices run over all d.o.f. As discussed above, terms on the right-hand side such as $D_{\mathbf{A A}}(p) D_{\mathbf{A A}}(k-p)$ (and quark loops if any), where both factors are noninstantaneous, do not contribute to these SD equations. 

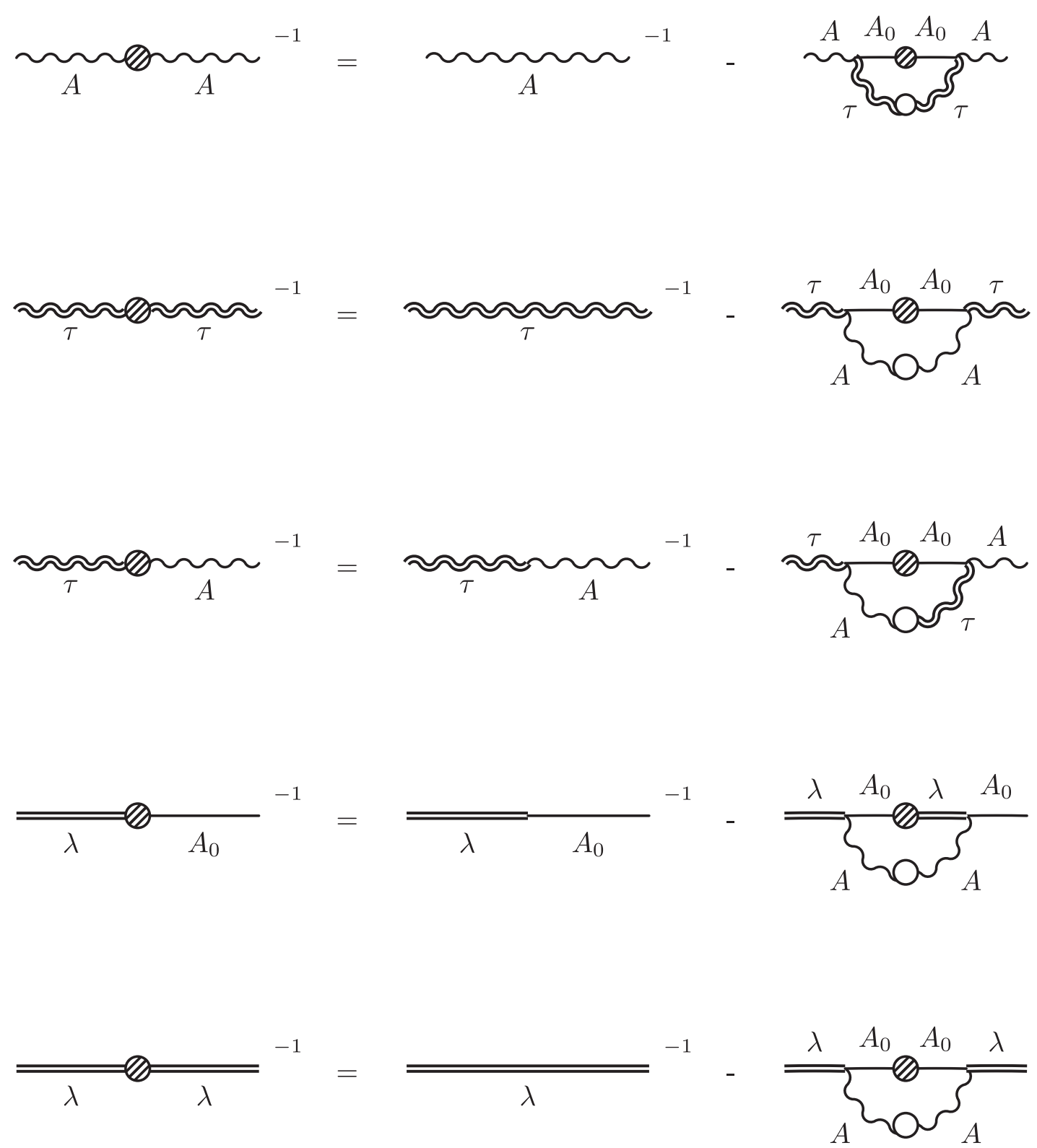

FIG. 2. Diagrammatic representation of the SDE. The shaded circle represents an instantaneous propagator and the empty circle an equal-time propagator. The instantaneous propagators are straight lines and the equal-time propagators are wavy.

As follows from Eq. (5.2), we now set $D_{\lambda \lambda}=0$ on the right-hand side of the SD equations where it appears, namely in the first term of the second line of Eq. (5.4). The remaining two terms in the second line of Eq. (5.4) appear to suffer from terrible divergences. In position space, each is the product of two instantaneous propagators. For example the second term in that line is $\delta^{2}\left(x_{0}-y_{0}\right) D_{\lambda A_{0}}(\mathbf{x}-\mathbf{y}) D_{\lambda A_{0}}(\mathbf{x}-\mathbf{y})$. The second and third terms contain the divergent integral $\int d p_{0}=\infty$, which is the momentum-space manifestation of the divergent factor $\delta^{2}\left(x_{0}-y_{0}\right)$. These are the famous energy divergences of the Coulomb gauge which cancel between the second and third term [36-39],

$$
\int d p_{0}\left(D_{A_{0} \lambda}(\mathbf{p}) D_{A_{0} \lambda}(\mathbf{k}-\mathbf{p})+D_{c \bar{c}}(\mathbf{p}) D_{c \bar{c}}(\mathbf{k}-\mathbf{p})\right)=0
$$

by virtue of $D_{c \bar{c}}=i D_{A_{0} \lambda}$, Eq. (5.2). Thus, all three terms in the second line in Eq. (5.4) are conveniently eliminated. (This argument is not rigorous because there remain unresolved ambiguities in the Coulomb gauge [37].) Each of the remaining terms in the SDE is the product of an instantaneous propagator and an equal-time propagator, which together give a finite instantaneous contribution. 
With these results the DSE simplifies to

$$
\begin{aligned}
\Gamma_{\mathbf{A A}}(\mathbf{k})= & \mathbf{k}^{2}+\frac{N g^{2}}{(2 \pi)^{d+1}} \int d^{d+1} p \frac{d-2+(\hat{k} \cdot \hat{p})^{2}}{d-1} \\
& \times D_{\tau \tau}(p) D_{A_{0} A_{0}}(\mathbf{k}-\mathbf{p}) \\
\Gamma_{\tau \tau}(\mathbf{k})= & 1+\frac{N g^{2}}{(2 \pi)^{d+1}} \int d^{d+1} p \frac{d-2+(\hat{k} \cdot \hat{p})^{2}}{d-1} \\
& \times D_{\mathbf{A A}}(p) D_{A_{0} A_{0}}(\mathbf{k}-\mathbf{p}) \\
\Gamma_{\tau \mathbf{A}}(k)= & -k_{0}+\frac{N g^{2}}{(2 \pi)^{d+1}} \int d^{d+1} p \frac{d-2+(\hat{k} \cdot \hat{p})^{2}}{d-1} \\
& \times D_{\mathbf{A} \tau}(p) D_{A_{0} A_{0}}(\mathbf{k}-\mathbf{p}) \\
\Gamma_{A_{0} A_{0}}(\mathbf{k})= & 0 \\
\Gamma_{\lambda \lambda}(\mathbf{k})= & \mathbf{k}^{2}+\frac{N g^{2} \mathbf{k}^{2}}{(2 \pi)^{d+1}} \int d^{d+1} p\left[1-(\hat{k} \cdot \hat{p})^{2}\right] \\
& \times D_{\mathbf{A A}}(p) D_{A_{0} A_{0}}(\mathbf{k}-\mathbf{p}) \\
& \times D_{\mathbf{A A}}(p) D_{A_{0} \lambda}(\mathbf{k}-\mathbf{p}) . \\
\Gamma_{\lambda A_{0}}(\mathbf{k})= & i \mathbf{k}^{2}+\frac{N g^{2} \mathbf{k}^{2}}{(2 \pi)^{d+1}} \int d^{d+1} p\left[1-(\hat{k} \cdot \hat{p})^{2}\right] \\
&
\end{aligned}
$$

\section{EQUAL-TIME PROPAGATOR FROM THE LOOP INTEGRAL}

Consider the loop integral for $\Gamma_{\mathbf{A A}}(\mathbf{k})$. The only appearance of $p_{0}$ in the integrand occurs in $D_{\tau \tau}\left(p_{0}, \mathbf{p}\right)$, so the loop integral over $p_{0}$ takes the form

$$
\int \frac{d p_{0}}{2 \pi} D_{\tau \tau}\left(p_{0}, \mathbf{p}\right)=D_{\tau \tau}^{E T}(\mathbf{p}),
$$

where the right-hand side is the equal-time propagator. Indeed, it is a special case of the fourier transform,

$$
\tilde{D}_{\tau \tau}(t, \mathbf{p})=\int \frac{d p_{0}}{2 \pi} \exp \left(i p_{0} t\right) D_{\tau \tau}\left(p_{0}, \mathbf{p}\right)
$$

at $t=0, D_{\tau \tau}^{E T}(\mathbf{p})=\tilde{D}_{\tau \tau}(0, \mathbf{p})$. The remaining integration $\int d^{d} p$ is an integral over the space dimension $d$. The same is true for all the loop integrals.

We now show that

$$
\Gamma_{\tau \mathbf{A}}\left(k_{0}, \mathbf{k}\right)=-k_{0}
$$

is a solution of Eq. (5.15). Indeed, suppose this is true. It gives

$$
\begin{aligned}
& \left(\begin{array}{cc}
D_{\tau \tau}\left(k_{0}, \mathbf{k}\right) & D_{\tau \mathbf{A}}\left(k_{0}, \mathbf{k}\right) \\
D_{\mathbf{A} \tau}\left(k_{0}, \mathbf{k}\right) & D_{\mathbf{A A}}\left(k_{0}, \mathbf{k}\right)
\end{array}\right) \\
& =\left(\begin{array}{cc}
\Gamma_{\tau \tau}(\mathbf{k}) & -k_{0} \\
k_{0} & \Gamma_{\mathbf{A A}}(\mathbf{k})
\end{array}\right)^{-1} \\
& =\frac{1}{k_{0}^{2}+\Gamma_{\tau \tau}(\mathbf{k}) \Gamma_{\mathbf{A A}}(\mathbf{k})}\left(\begin{array}{cc}
\Gamma_{\mathbf{A A}}(\mathbf{k}) & k_{0} \\
-k_{0} & \Gamma_{\tau \tau}(\mathbf{k})
\end{array}\right) \text {, }
\end{aligned}
$$

which implies

$$
\int \frac{d p_{0}}{2 \pi} D_{\mathbf{A} \tau}\left(p_{0}, \mathbf{p}\right)=0
$$

because $D_{\mathbf{A} \tau}\left(p_{0}, \mathbf{p}\right)$ is odd in $p_{0}$. It follows that the integral in Eq. (5.15) vanishes, which gives $\Gamma_{\tau \mathbf{A}}\left(k_{0}, \mathbf{k}\right)=-k_{0}$ so Eq. (5.15) is satisfied. (There may also be a nonperturbative solution which cannot be expressed as a power series in $\mathrm{g}$.)

The SD equations now read

$$
\begin{aligned}
\Gamma_{\mathbf{A A}}(\mathbf{k})= & \mathbf{k}^{2}+\frac{N g^{2}}{(2 \pi)^{d}} \int d^{d} p \frac{d-2+(\hat{k} \cdot \hat{p})^{2}}{d-1} \\
& \times D_{\tau \tau}^{E T}(\mathbf{p}) D_{A_{0} A_{0}}(k-p) \\
\Gamma_{\tau \tau}(\mathbf{k})= & 1+\frac{N g^{2}}{(2 \pi)^{d}} \int d^{d} p \frac{d-2+(\hat{k} \cdot \hat{p})^{2}}{d-1} \\
& \times D_{\mathbf{A A}}^{E T}(\mathbf{p}) D_{A_{0} A_{0}}(\mathbf{k}-\mathbf{p}) \\
\Gamma_{\tau \mathbf{A}}(k)= & -k_{0} \\
\Gamma_{A_{0} A_{0}}(\mathbf{k})= & 0 \\
\Gamma_{\lambda \lambda}(\mathbf{k})= & \mathbf{k}^{2}+\frac{N g^{2} \mathbf{k}^{2}}{(2 \pi)^{d}} \int d^{d} p\left[1-(\hat{k} \cdot \hat{p})^{2}\right] \\
& \times D_{\mathbf{A A}}^{E T}(\mathbf{p}) D_{A_{0} A_{0}}(\mathbf{k}-\mathbf{p}) \\
\Gamma_{\lambda A_{0}}(\mathbf{k})= & i \mathbf{k}^{2}+\frac{N g^{2} \mathbf{k}^{2}}{(2 \pi)^{d}} \int d^{d} p\left[1-(\hat{k} \cdot \hat{p})^{2}\right] \\
& \times D_{\mathbf{A} \mathbf{A}}^{E T}(\mathbf{p}) D_{A_{0} \lambda}(\mathbf{k}-\mathbf{p}) .
\end{aligned}
$$

Upon close inspection of Eq. (6.4), something may seem amiss. The $k_{0}$ dependence in the propagators implies that these propagators are noninstantaneous, which violates the symmetry discussed in Sec. III. A brief calculation with a power-law ansatz is provided in Appendix $\mathrm{C}$ to show that in the infrared limit, this symmetry is restored, exhibiting a remarkable self consistency of the approach.

\section{REDUCTION TO THREE UNKNOWNS}

The propagators and inverse propagators, $D_{r s} \Gamma_{s t}=\delta_{r t}$, of the scalar fields are related by 


$$
\begin{aligned}
& \left(\begin{array}{cc}
D_{\lambda \lambda}(\mathbf{k}) & D_{\lambda A_{0}}(\mathbf{k}) \\
D_{A_{0} \lambda}(\mathbf{k}) & D_{A_{0} A_{0}}(\mathbf{k})
\end{array}\right) \\
& \quad=\left(\begin{array}{cc}
\Gamma_{\lambda \lambda}(\mathbf{k}) & \Gamma_{\lambda A_{0}}(\mathbf{k}) \\
\Gamma_{A_{0} \lambda}(\mathbf{k}) & \Gamma_{A_{0} A_{0}}(\mathbf{k})
\end{array}\right)-1 \\
& =\left(\begin{array}{cc}
0 & {\left[\Gamma_{A_{0} \lambda}(\mathbf{k})\right]^{-1}} \\
{\left[\Gamma_{\lambda A_{0}}(\mathbf{k})\right]^{-1}} & -\Gamma_{\lambda \lambda}(\mathbf{k})\left[\Gamma_{\lambda A_{0}}(\mathbf{k})\right]^{-2}
\end{array}\right),
\end{aligned}
$$

where we have used $\Gamma_{A_{0} A_{0}}(\mathbf{k})=0$. This gives $D_{\lambda \lambda}(\mathbf{k})=0$, in accordance with Eq. (5.2).

Correspondingly for the dynamical propagators, we have Eq. (6.4), which gives for the equal-time propagators

$$
\begin{aligned}
&\left(\begin{array}{cc}
D_{\tau \tau}^{E T}(\mathbf{k}) & D_{\tau \mathbf{A}}^{E T}(\mathbf{k}) \\
D_{\mathbf{A} \tau}^{E T}(\mathbf{k}) & D_{\mathbf{A A}}^{E T}(\mathbf{k})
\end{array}\right)= \int \frac{d k_{0}}{2 \pi} \frac{1}{k_{0}^{2}+\Gamma_{\tau \tau}(\mathbf{k}) \Gamma_{\mathbf{A A}}(\mathbf{k})} \\
& \times\left(\begin{array}{cc}
\Gamma_{\mathbf{A A}}(\mathbf{k}) & k_{0} \\
-k_{0} & \Gamma_{\tau \tau}(\mathbf{k})
\end{array}\right) \\
&\left(\begin{array}{cc}
D_{\tau \tau}^{E T}(\mathbf{k}) & D_{\tau \mathbf{A}}^{E T}(\mathbf{k}) \\
D_{\mathbf{A} \tau}^{E T}(\mathbf{k}) & D_{\mathbf{A A}}^{E T}(\mathbf{k})
\end{array}\right) \\
&=\frac{1}{2}\left(\begin{array}{cc}
\left(\Gamma_{\mathbf{A A}} / \Gamma_{\tau \tau}\right)^{1 / 2}(\mathbf{k}) & 0 \\
0 & \left(\Gamma_{\tau \tau} / \Gamma_{\mathbf{A A}}\right)^{1 / 2}(\mathbf{k})
\end{array}\right) .
\end{aligned}
$$

Note that $D_{\tau \mathbf{A}}\left(k_{0}, \mathbf{k}\right)$ is odd in $k_{0}$ which gives $D_{\tau \mathbf{A}}^{E T}(\mathbf{k})=$ $\int d k_{0} D_{\tau \mathbf{A}}\left(k_{0}, \mathbf{k}\right)=0$, as claimed.

From the last equation we have the simple identity,

$$
4 D_{\tau \tau}^{E T}(\mathbf{k}) D_{\mathbf{A A}}^{E T}(\mathbf{k})=1,
$$

which determines $D_{\tau \tau}^{E T}$. There remain only three independent unknown functions $D_{\mathbf{A A}}^{E T}(\mathbf{k}), D_{A_{0} A_{0}}(\mathbf{k})$ and $D_{A_{0} \lambda}(\mathbf{k})$. We also have from Eq. (7.3),

$$
\left[D_{\mathbf{A A}}^{E T}(\mathbf{k})\right]^{2}=\frac{\Gamma_{\tau \tau}(\mathbf{k})}{4 \Gamma_{\mathbf{A A}}(\mathbf{k})} .
$$

The last two equations give

$$
\frac{\Gamma_{\mathbf{A A}}(\mathbf{k})}{D_{\tau \tau}^{E T}(\mathbf{k})}=\frac{\Gamma_{\tau \tau}(\mathbf{k})}{D_{\mathbf{A A}}^{E T}(\mathbf{k})} .
$$

We now substitute the right-hand side of the SDE for $\Gamma_{\mathbf{A A}}(\mathbf{k})$ and $\Gamma_{\tau \tau}(\mathbf{k})$, Eqs. (6.6) and (6.7), into the last equation, which gives

$$
\begin{aligned}
4 \mathbf{k}^{2} & D_{\mathbf{A A}}^{E T}(\mathbf{k})-\left[D_{\mathbf{A A}}^{E T}(\mathbf{k})\right]^{-1} \\
= & g^{2} N \int \frac{d^{d} p}{(2 \pi)^{d}} \frac{d-2+(\hat{p} \cdot \hat{k})^{2}}{d-1}\left(\frac{D_{\mathbf{A A}}^{E T}(\mathbf{p})}{D_{\mathbf{A A}}^{E T}(\mathbf{k})}-\frac{D_{\mathbf{A A}}^{E T}(\mathbf{k})}{D_{\mathbf{A A}}^{E T}(\mathbf{p})}\right) \\
& \times D_{A_{0} A_{0}}(\mathbf{p}-\mathbf{k})
\end{aligned}
$$

$$
\begin{aligned}
& D_{A_{0} A_{0}}(\mathbf{k})\left[D_{c \bar{c}}(\mathbf{k})\right]^{-2} \\
& =\mathbf{k}^{2}+\frac{N g^{2} \mathbf{k}^{2}}{(2 \pi)^{d}} \int d^{d} p\left[1-(\hat{k} \cdot \hat{p})^{2}\right] D_{\mathbf{A} \mathbf{A}}^{E T}(\mathbf{p}) D_{A_{0} A_{0}}(\mathbf{k}-\mathbf{p}) \\
& {\left[D_{c \bar{c}}(\mathbf{k})\right]^{-1}=} \\
& \quad \mathbf{k}^{2}-\frac{N g^{2} \mathbf{k}^{2}}{(2 \pi)^{d}} \int d^{d} p\left[1-(\hat{k} \cdot \hat{p})^{2}\right] \\
& \quad \times D_{\mathbf{A A}}^{E T}(\mathbf{p}) D_{c \bar{c}}(\mathbf{k}-\mathbf{p})
\end{aligned}
$$

where the last two equations come from Eqs. (6.10) and (6.11), and we have used

$$
\Gamma_{\lambda A_{0}}=i \Gamma_{\bar{c} c}=i\left[D_{c \bar{c}}\right]^{-1}=\left[D_{\lambda A_{0}}\right]^{-1} .
$$

Altogether there are three equations for the three propagators $D_{\mathbf{A A}}^{E T}(\mathbf{k}), D_{c \bar{c}}(\mathbf{k})$ and $D_{A_{0} A_{0}}(\mathbf{k})$. These three quantities are invariant under the remnant gauge symmetry $g(t)$. Suppose the three equations are solved, so these three quantities are known. Then one can recover a fourth quantity, $D_{\tau \tau}^{E T}(\mathbf{k})$, from $4 D_{\tau \tau}^{E T}(\mathbf{k}) D_{\mathbf{A A}}^{E T}(\mathbf{k})=1$. These four quantities are all that appear on the right-hand side of Eqs. (6.6) through (6.11), from which one can recover all $\Gamma_{\alpha \beta}$ and hence all propagators $D_{\beta \gamma}$.

\section{GAUGE CONDITION ON THE LATTICE AND IN THE CONTINUUM}

Beside imposing the Coulomb gauge condition, $\partial_{i} A_{i}=0$, we must also address the nonperturbative issue of Gribov copies [40,41], [42,43].

A gauge choice that is accessible to numerical simulation is implemented by minimizing (the lattice analog of) the spatial Hilbert norm,

$$
F_{A}(g) \equiv \int d^{d+1} x \sum_{i=1}^{d}\left({ }^{g} A_{i}^{b}\right)^{2},
$$

with respect to gauge transformations $g(x)$, where $A_{\mu}=$ $\frac{1}{2} i \tau^{b} A_{\mu}^{b}$ and ${ }^{g} A_{\mu}=g^{-1} A_{\mu} g+g^{-1} \partial_{\mu} g$. At a global or local minimum, the gauge condition $\partial_{i} A_{i}=0$ is satisfied, and all eigenvalues of the Faddeev-Popov operator $M(A)$ are nonnegative $\lambda_{n}(g A) \geq 0$. The set of continuum configurations that satisfy these conditions is designated by $\Omega$ and is called the "(first) Gribov region." It is a convex region in configuration space (A-space) that is bounded in every direction. Its boundary, $\partial \Omega$, is called the "Gribov horizon." At large volume $V, \Omega$ is specified by $H(g A) \leq\left(N^{2}-1\right) d V$, where the "horizon function," $H(g A)$, is defined in Eq. (A2) [44]. The actual lattice simulation with which we shall compare was gauge-fixed by finding one local minimum of the minimizing functional for each gauge orbit. ${ }^{3}$

\footnotetext{
${ }^{3}$ There are various gauge choices possible within $\Omega$.
} 
The set $\Lambda$ of absolute minima of the minimizing functional provides a complete gauge fixing. It would be nice if we could perform the (functional) integral over $\Lambda$, but we cannot, because we do not have an explicit description of $\Lambda$ in the physical limit of large volume $V$, as we do for $\Omega .{ }^{4}$ In this situation, we make the approximation which consists in integrating over $\Omega$ instead of $\Lambda$. This approximation introduces a certain "gauge-fixing error," and the total error of the present calculation is the compound of this gauge-fixing error with the error introduced by the truncation of terms in the SDE.

In the limit of large volume $V$, the functional integral over the Gribov region $\Omega$ gets concentrated on its surface $\partial \Omega,{ }^{5}$ and the cut-off at the Gribov horizon is replaced by insertion of the factor $\delta\left[\left(N^{2}-1\right) d V-H\right]$, which enforces the "horizon condition." "In Appendix A, it is shown that the horizon condition $\langle H\rangle=\left(N^{2}-1\right) d V$, and the maximum- $b$ condition, $\lim _{|\mathbf{k}| \rightarrow \mathbf{0}} b(\mathbf{k})=\infty$, are equivalent, where $b(\mathbf{k}) \equiv \mathbf{k}^{2} D_{c \bar{c}}(\mathbf{k})$ is the ghost dressing function. ${ }^{7}$

The maximum- $b$ condition states that the ghost propagator is of longer range than the electrostatic potential, which is the same as requiring that the ghost propagator $D_{c \bar{c}}(\mathbf{k})$ be more singular than $\frac{1}{\mathbf{k}^{2}}$ at $\mathbf{k}=0$, or equivalently that the inverse ghost propagator $\Gamma_{\bar{c} c}(\mathbf{k})=D_{c \bar{c}}^{-1}(\mathbf{k})$ vanish more rapidly than $\mathbf{k}^{2}$. This is done by subtracting the term of order $\mathbf{k}^{2}$ on the right-hand side of the SD equation for $\Gamma_{\bar{c} c}$, so it reads

$$
\begin{aligned}
\Gamma_{\bar{c} c}(\mathbf{k})= & {\left[D_{c \bar{c}}(\mathbf{k})\right]^{-1} } \\
= & -g^{2} N \mathbf{k}^{2} \int \frac{d^{d} p}{(2 \pi)^{d}}\left[1-(\hat{p} \cdot \hat{k})^{2}\right] \\
& \times D_{A A}^{E T}(\mathbf{p})\left[D_{c \bar{c}}(\mathbf{p}+\mathbf{k})-D_{c \bar{c}}(\mathbf{p})\right] .
\end{aligned}
$$

There is an overall coefficient $\mathbf{k}^{2}$, and the integrand vanishes at $\mathbf{k}=0$, so the right-hand side vanishes faster

\footnotetext{
${ }^{4}$ However it is known (a) that $\Lambda$ is also a bounded, convex region, which is contained in the Gribov region, $\Lambda \subset \Omega$, (b) that part of the boundary of $\Lambda$ coincides with part of the boundary of $\Omega$, and (c) that there are relative minima that are Gribov copies inside $\Omega$.

${ }^{5}$ This is easily understood. The integral over a unit ball $0 \leq r \leq 1$ in a space of dimension $N$ has radial measure $r^{N-1} d r$. In the limit of large $N \rightarrow \infty$, the radial measure gets concentrated on the surface $\delta(1-r)$.

${ }^{6}$ This can be converted to a local action, at the cost of introducing additional bose and fermi ghosts [45].

${ }^{7}$ Observe that for all the different values of $\mathbf{k}$, the corresponding eigenvalues all change sign at the same surface $\langle H(g A)\rangle=\left(N^{2}-1\right) d V$. This has been called "all horizons are one horizon" [45]. This analytic result is consistent with lattice study in Coulomb gauge of Nakagawa et al. [46] who state "Our result is consistent with the hypothesis in the Gribov-Zwanziger scenario that the measure of the path integral is concentrated on the part of the horizon where "all horizons are one horizon." This has also been observed in Landau gauge [47].
}

than $\mathbf{k}^{2}$. It is not obvious whether the last integral is positive for all $\mathbf{k}$, as it should be if $M(\mathbf{A})$ is a positive matrix, so it is a nice check that when it is evaluated below, $I(\alpha, \gamma)$, given in Eq. (10.7), it is in fact positive.

\section{THREE EQUATIONS FOR THREE CRITICAL EXPONENTS}

\section{A. First SD equation for critical exponents}

We now assume that the propagators approach an asymptotic limit at small $k=|\mathbf{k}|$ which is a power law, with critical exponents defined in Table I. We substitute this power-law ansatz into Eq. (7.7),

$$
\frac{4 k^{2} c}{k^{\gamma}}-\frac{g^{2} k^{\gamma}}{c}=N \int \frac{d^{d} p}{(2 \pi)^{d}} \frac{d-2+(\hat{p} \cdot \hat{k})^{2}}{d-1}\left(\frac{k^{\gamma}}{p^{\gamma}}-\frac{p^{\gamma}}{k^{\gamma}}\right) \frac{\bar{d}}{|\vec{p}-\mathbf{k}|^{\delta}} .
$$

For this to yield a bona fide solution, the loop integral must converge. There is a singularity due to the colorCoulomb potential, $1 /|\mathbf{p}-\mathbf{k}|^{\delta}$. However the factor $\frac{k^{\gamma}}{p^{\gamma}}-\frac{p^{\gamma}}{k^{\gamma}}$ vanishes at $\mathbf{p}=\mathbf{k}$ like $(\mathbf{p}-\mathbf{k})^{2}$, so the integral converges at $\mathbf{p}=\mathbf{k}$ provided $\delta<d+2$. There is a singularity at $p=0$ due to the terms $p^{\gamma}$ and $p^{-\gamma}$, so the integral does not converge at $p=0$ unless $d>|\gamma|$. The loop integral must also converge at high $p$. Suppose $\gamma$ is positive $\gamma>0$. In this case, the highest power of $p$ in the last integrand comes from the power $p^{\gamma}$ in the second term in the parenthesis, and the integral will not converge at high $p$ unless $d+\gamma<\delta$. Now suppose instead that $\gamma$ is negative, $\gamma<0$. In this case, the highest power of $p$ comes from the first term in parenthesis $1 / p^{\gamma}$, and the loop integral will not converge unless $d-\gamma<\delta$, and we have established,

$$
d<d+|\gamma|<\delta<d+2
$$

By power-counting one sees that the right-hand side of Eq. (9.1) is proportional to $k^{d-\delta}$. The inequalites just obtained imply that in the infrared asymptotic limit, $k \rightarrow 0$, the right-hand side is dominant over each term on the left-hand side. Indeed, it dominates the first term in this limit provided $\delta-d>\gamma-2$, that is, if $\delta>d+\gamma-2$, which holds by virtue of Eq. (9.2). Likewise it dominates the second term on the left provided $\delta-d>-\gamma$, that is, if $\delta>d-\gamma$, which is also true. Therefore in the infrared asymptotic limit only the right-hand side survives, and the first SDE reads,

TABLE I. Critical exponents defined in the infrared asymptotic limit.

$$
\begin{aligned}
D_{c \bar{c}} & \Rightarrow a k^{-\alpha} \\
g^{2} D_{\mathbf{A A}}^{E T} & \Rightarrow c k^{-\gamma} \\
g^{2} D_{A_{0} A_{0}} & \Rightarrow \bar{d} k^{-\delta}
\end{aligned}
$$


$0=\int \frac{d^{d} p}{(2 \pi)^{d}} \frac{d-2+(\hat{p} \cdot \hat{k})^{2}}{d-1}\left(\frac{k^{\gamma}}{p^{\gamma}}-\frac{p^{\gamma}}{k^{\gamma}}\right) \frac{\bar{d}}{|\mathbf{p}-\mathbf{k}|^{\delta}}$.

The inequality $\delta>d$, just derived, is none other than the condition in space dimension $d$, for the color-Coulomb potential to be confining, $\lim _{r \rightarrow \infty} V_{C}(r)=\infty$, for we have ${ }^{8}$

$$
\begin{aligned}
V_{C}(r) & =\int d^{d} p(2 \pi)^{-d} \exp (i \mathbf{p} \cdot \mathbf{x}) D_{A_{0} A_{0}}(\mathbf{p}) \\
& \sim \int d^{d} p \exp (i \mathbf{p} \cdot \mathbf{x}) / p^{\delta} \sim r^{\delta-d} .
\end{aligned}
$$

Thus, Eq. (9.3) is a sufficient condition for the colorCoulomb potential to be confining.

\section{B. Second SD equation for critical exponents}

Insertion of the power laws into (8.2) yields

$$
\begin{aligned}
\Gamma_{\bar{c} c}(\mathbf{k}) & =\left[D_{c \bar{c}}(\mathbf{k})\right]^{-1} \\
\Rightarrow \frac{k^{\alpha}}{a} & =-N \mathbf{k}^{2} \int \frac{d^{d} p}{(2 \pi)^{d}}\left[1-(\hat{p} \cdot \hat{k})^{2}\right] \frac{c}{|\mathbf{p}|^{\gamma}}\left(\frac{a}{|\mathbf{p}-\mathbf{k}|^{\alpha}}-\frac{a}{|\mathbf{p}|^{\alpha}}\right) .
\end{aligned}
$$

By counting powers of $k$ and $p$ on the left- and right-hand sides, we obtain $\alpha=2+d-\gamma-\alpha$, which gives the "sum rule"

$$
2 \alpha+\gamma=d+2
$$

Moreover, the right-hand side has a coefficient $\mathbf{k}^{2}$, and an integrand that vanishes with $\mathbf{k}$, so the right-hand side vanishes with $k$ more rapidly than $\mathbf{k}^{2}$. We conclude that $\alpha>2$, so the ghost propagator is more singular than the free propagator. ${ }^{9}$ This was imposed by the horizon condition. We require that the loop integral (9.5) converges at high $p$. This yields the inequality $d<\gamma+\alpha+2$. Indeed, the subtraction term cancels the leading term in $1 / p$ at high $p$, and the next power is killed by angular integration, so the subtraction term increases the power of $1 / p$ by 2 . We substitute

$$
\gamma=d+2-2 \alpha
$$

into the last inequality and obtain $\alpha<4$ and, thus,

$$
2<\alpha<4
$$

\footnotetext{
${ }^{8}$ A linearly rising color-Coulomb potential, which is favored by lattice calculations in Coulomb gauge [48], corresponds to $\delta=d+1$.

${ }^{9}$ The case $d=2$ is singular. We deal with this by continuing in dimension to $d>2$, and for the case $d=2$, we take the limit $d \rightarrow 2$ at the end of the calculation.
}

\section{Third SD equation for critical exponents}

Upon insertion of the power ansatz into Eq. (7.8) we obtain

$$
\Gamma_{\lambda \lambda}(k) \Rightarrow \frac{\bar{d} k^{2 \alpha}}{a^{2} k^{\delta}}=k^{2}+N \int \frac{d^{d} p}{(2 \pi)^{d}} \frac{p^{2} k^{2}-(p \cdot k)^{2}}{p^{2}} \frac{c}{|\mathbf{p}|^{\gamma}} \frac{\bar{d}}{|\mathbf{p}-\mathbf{k}|^{\delta}}
$$

The integral converges provided $d-\gamma-\delta<0$, which agrees with the confinement bound, Eq. (9.2). In this case, the tree-level term is negligible compared to the loop term in the infrared asymptotic limit, and this ISD simplifies to

$$
\frac{k^{d+2-\gamma-\delta}}{a^{2}}=N \int \frac{d^{d} p}{(2 \pi)^{d}} \frac{p^{2} k^{2}-(p \cdot k)^{2}}{p^{2}} \frac{c}{|\mathbf{p}|^{\gamma}} \frac{1}{|\mathbf{p}-\mathbf{k}|^{\delta}} .
$$

\section{DETERMINATION OF THE INFRARED CRITICAL EXPONENTS}

From Eq. (9.5), we obtain

$$
\frac{1}{a^{2} c}=I(\alpha, \gamma)
$$

where

$$
\begin{aligned}
I(\alpha, \gamma) \equiv & -N|\mathbf{k}|^{\alpha+\gamma-d} \int \frac{d^{d} p}{(2 \pi)^{d}}\left[1-(\hat{p} \cdot \hat{k})^{2}\right] \\
& \times \frac{1}{|\mathbf{p}|^{\gamma}}\left(\frac{1}{|\mathbf{p}+\mathbf{k}|^{\alpha}}-\frac{1}{|\mathbf{p}|^{\alpha}}\right) .
\end{aligned}
$$

Likewise from Eq. (9.10) we obtain

$$
\frac{1}{a^{2} c}=L(\delta, \gamma)
$$

where

$L(\delta, \gamma) \equiv N|\mathbf{k}|^{\delta+\gamma-d} \int \frac{d^{d} p}{(2 \pi)^{d}}\left[1-(\hat{p} \cdot \hat{k})^{2}\right] \frac{1}{|\mathbf{p}|^{\gamma}} \frac{1}{|\mathbf{p}+\mathbf{k}|^{\delta}}$

which gives

$$
I(\alpha, \gamma)=L(\delta, \gamma) .
$$

One has

$$
\begin{aligned}
L(\delta, \gamma)= & \frac{N(d-1)}{2(4 \pi)^{d / 2}} \\
& \times \frac{\Gamma[(\delta+\gamma-d) / 2] \Gamma[(d+2-\delta) / 2] \Gamma[(d-\gamma) / 2]}{\Gamma[(\gamma+2) / 2] \Gamma[\delta / 2] \Gamma[(2 d+2-\delta-\gamma) / 2]},
\end{aligned}
$$


The integral $I(\alpha, \gamma)$ was evaluated in (A.17) of [49], with $I(\alpha)=I_{G}\left(\alpha_{G}\right)$ and $\alpha=2+2 \alpha_{G}$, with the result ${ }^{10}$

$$
\begin{aligned}
I(\alpha, \gamma)= & -L(\alpha, \gamma) \\
= & -\frac{N(d-1)}{2(4 \pi)^{d / 2}} \\
& \times \frac{\Gamma[(\alpha+\gamma-d) / 2] \Gamma[(d+2-\alpha) / 2] \Gamma[(d-\gamma) / 2]}{\Gamma[(\gamma+2) / 2] \Gamma[\alpha / 2] \Gamma[(2 d+2-\alpha-\gamma) / 2]} .
\end{aligned}
$$

$[I(\alpha, \gamma)$ is positive because $\alpha+\gamma-d=2-\alpha<0$, provided that $\alpha<4$, which holds by Eq. (9.8).] We have

$$
-L(\alpha, \gamma)=L(\delta, \gamma)
$$

which gives

$$
\begin{aligned}
& \frac{\Gamma[(\delta+\gamma-d) / 2] \Gamma[(d+2-\delta) / 2]}{\Gamma[\delta / 2] \Gamma[(2 d+2-\delta-\gamma) / 2]} \\
& \quad=-\frac{\Gamma[(\alpha+\gamma-d) / 2] \Gamma[(d+2-\alpha) / 2]}{\Gamma[\alpha / 2] \Gamma[(2 d+2-\alpha-\gamma) / 2]} .
\end{aligned}
$$

A third relation between the critical exponents is provided by Eq. (9.3). An obvious solution to that equation is provided by

$$
\gamma=0
$$

We have searched diligently for another solution, but we have not found any. This solution and the sum rule (9.7) then give

$$
\alpha=\frac{1}{2}(d+2),
$$

and we finally obtain the following condition, expressed with a new parameter, $\theta \equiv \delta-d-1$,

$$
E(\theta, d)=F(d)
$$

where

$$
\begin{aligned}
E(\theta, d) & \equiv \frac{\pi}{\cos \left(\frac{\pi \theta}{2}\right) \Gamma\left(\frac{d+1+\theta}{2}\right) \Gamma\left(\frac{d+1-\theta}{2}\right)} \\
F(d) & \equiv-\frac{\Gamma\left(\frac{2-d}{4}\right)}{\Gamma\left(\frac{3 d+2}{4}\right)} .
\end{aligned}
$$

We have used $\Gamma(x) \Gamma(1-x)=\pi / \sin (\pi x)$. The change of variable from $\delta$ to $\theta$ is convenient because $E(\theta, d)$ is even in $\theta, E(\theta, d)=E(-\theta, d)$.

\footnotetext{
${ }^{10}$ This result is the same as ignoring the subtraction term in (10.2) and continuing $L(\delta, \gamma)$ analytically from $\delta$ to $\alpha$, at fixed $\gamma$.
}

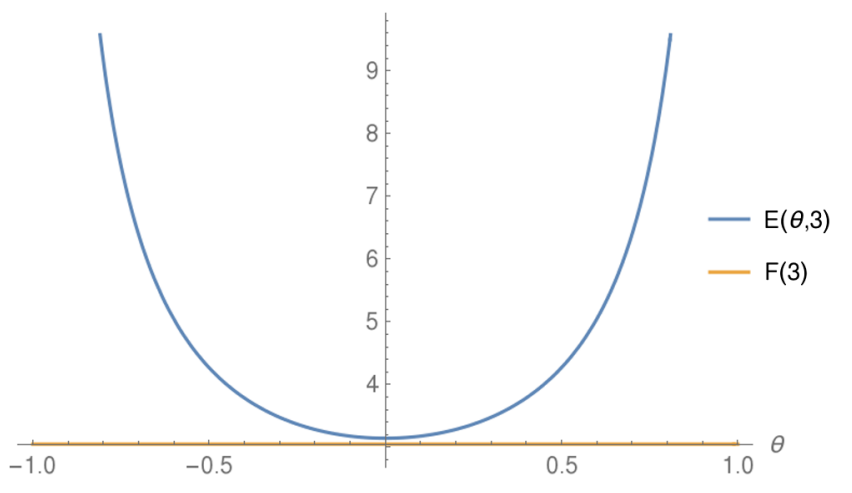

FIG. 3. A near miss at space dimension $d=3$. As $d$ decreases below $d_{c}$, the curved line intersects the straight horizontal line twice.

For a given space dimension $d$, let $\theta(d)$ be a solution to Eq. (10.12), then the critical exponent of the colorCoulomb potential $\delta$ is recovered from

$$
\delta(d)=d+1+\theta(d)
$$

The function $E(\theta, d)$ is finite and positive for $\theta$ in the interval $-1<\theta<1$ (which corresponds to $d<\delta<d+2$ ), and is divergent at the end-points, $\theta= \pm 1$, where $\cos (\pi \theta / 2)$, which is in the denominator, vanishes, $\cos (\pi \theta / 2)=0$. Since $E(\theta, d)$ is even in $\theta$, if $\theta(d)$ is a solution to Eq. (10.12), then so is $-\theta(d)$, and the solutions to (10.12) form two branches $\theta_{+}(d)$ and $\theta_{-}(d)=-\theta_{+}(d)$ as shown in Fig. 3.

We are interested in integer space dimensions $d=2$ and $d=3$. However it is helpful to take $d$ to be a continuous variable in the interval $2<d<3$. The function $F(d)$ diverges in the limit $d \rightarrow 2$ which tells us that $\theta(2)= \pm 1$, for we have just seen that $E(\theta, d)$ is divergent at $\theta= \pm 1 .{ }^{11}$ These values of $\theta$ correspond to $\delta(d=2)=d+1 \pm 1=3 \pm 1$, so for $d=2$, there are two solutions $\delta_{-}(2)=2$ and $\delta_{+}(2)=4$. One sees in Fig. 4 that, as $d$ increases from $d=2$, the two branches, $\theta_{+}(d)$ and $\theta_{-}(d)$ approach each other monotonically, and at a critical dimension,

$$
d_{c}=2.9677 \ldots
$$

they merge at $\theta=0$, which corresponds to $\delta(\theta=0)=$ $d_{c}+1[14]$.

With the problem as stated, there is no solution for space dimension above the critical value $d>d_{c}$. However, 2.9677 is tantalizingly close to 3 , and it may be that there is a solution for $d=3$ (a) if the gauge-fixing error noted above were corrected, (b) if the truncation error were corrected, or (c) if perhaps the true asymptotic behavior

\footnotetext{
${ }^{11}$ The reader who wishes to avoid equating infinite quantities may prefer to solve the equation $[E(\theta, d)]^{-1}=[F(d)]^{-1}$.
} 


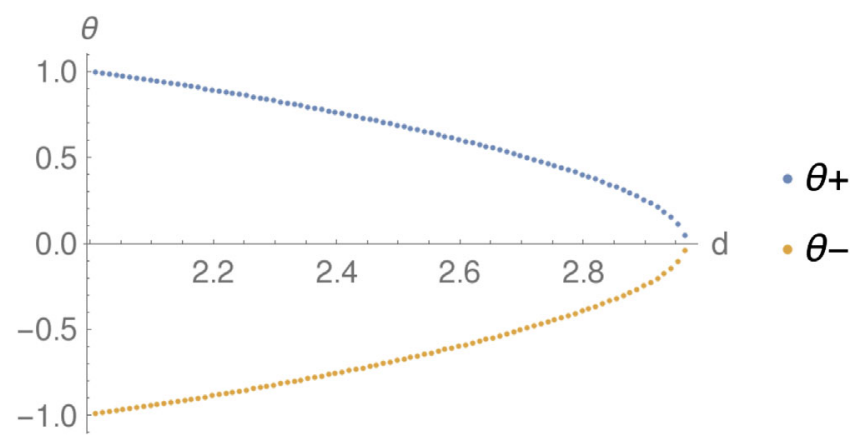

FIG. 4. Plot of the two solutions $\theta_{ \pm}(d)$. The critical exponent of the color-Coulomb potential is given by $\delta=d+1+\theta_{ \pm}(d)$.

isn't a pure power-law, but rather dominated by a power with multiplicative log corrections. We shall suppose one of these possibilities is in effect, and we consider that it is an approximation to replace the physical value $d=3$ by $d=d_{c}$, which is a difference of about $1 \%$. For $d=3$ and $\theta=0$, we have

$$
\begin{aligned}
E(\theta=0, d=3) & =\pi=3.14159265 \ldots \\
F(d=3) & =64 / 21=3.04761905 \ldots
\end{aligned}
$$

Instead of an equality, there is a difference of about $3 \%$.

The color-Coulomb potential,

$$
V_{\mathrm{C}}(r) \sim \int d^{d} k \exp (i k \cdot x) \frac{c}{k^{\delta}} \sim r^{\delta-d},
$$

is linear at large $r$ for $\delta=d+1$, and super-linear for $\delta>d+1$. As space dimension $d$ increases from $d=2$ to $d=d_{c}$, on the upper branch $\theta_{+}(d)$ decreases from $\theta_{+}(2)=1$ to $\theta_{+}\left(d_{c}\right)=0$ and is everywhere positive, $\theta_{+}(d) \geq 0$. It follows that $\delta(d)$, satisfies $\delta(d)=d+1+\theta_{+}(d) \geq d+1$. We conclude that on the upper branch the color-Coulomb potential $V_{\text {coul }}(R)$ is everywhere superlinear, except at $d=$ $d_{c}$ where it is linear. The lower branch is everywhere sublinear. $V_{\text {coul }}(R)$ is linear at the critical dimension, $d_{c}$, where $\delta\left(d_{c}\right)=d_{c}+1$. Thus, the upper branch accords with the exact theorem [50] which asserts that the colorCoulomb potential $V_{\text {coul }}(R)$ is bounded below by the gauge-invariant Wilson potential $V_{W}(R)$ that is linear at large $R$

$$
V_{\text {coul }}(R) \geq V_{W}(R)=\sigma R,
$$

whereas the lower branch does not. (Both branches accord with the exact bound $\delta>2 \alpha-2=d$ [48].)

The most natural choice between the two branches is to take the upper branch to be the physical solution because it satisfies the last inequality. In $d=2$ space dimensions, the calculation reported here yields, $\delta(d=2)=4$, which corresponds to a color-Coulomb potential that rises like $r^{2}$ at large $r$. This is an unexpectedly steep rise. One might speculate that the physical solution is a superposition or mixture of the two branches, so that the physical solution corresponds to a value of theta that lies between the two branches. If so, then $\theta$ must also satisfy $\theta \geq 0$, corresponding to $\delta \geq d+1$, to be consistent with the last inequality, and because linear rise corresponds to $\delta=d+1$. Finally we note that linear rise is energetically favorable compared to superlinear. A lattice calculation in two space dimensions (if it is not already in the literature) would throw some light on this matter.

In any case, the comparison we shall make with lattice gauge theory is with critical dimension $d_{c}=2.9677 \ldots$, which corresponds uniquely to linear rise, $\delta=d+1$.

\section{CONCLUSION}

\section{A. Comparison with lattice gauge theory}

Lattice calculations have been reported for SU(2) [28,29] and SU(3) [30]. In Table II, we compare our results with Langfeld and Moyaerts [28]. In the first column of Table II are the critical exponents, $\alpha, \gamma$, and $\delta$, of the propagators of the ghost, the spatial gluon, and the temporal gluon respectively, that are defined in Table I. In the second column, the values of these exponents found in the present article are expressed in terms of the dimension of space $d$. In the third column, the critical exponents defined in the present article are expressed, for the reader's convenience, in terms of the parameters defined by [28]. ( $\delta_{\mathrm{LM}}$ is the infrared exponent designated $\delta$ in [28].) The fourth column gives the numerical values of the critical exponents for the critical dimension found above, $d_{c}=2.9677 \ldots{ }^{12}$ The final column is result of the numerical simulation [28]. These authors do not give a numerical value for the infrared exponent of the transverse equal-time gluon propagator, $\gamma$, but state "At small momentum, the propagator becomes roughly momentum-independent and seems to approach a constant in the IR limit $|\mathbf{p}| \rightarrow 0$." This is consistent with our result, which gives for this infrared exponent, $\gamma=0$. If it is not accidental, the agreement between the fourth and the fifth column is remarkable for the accuracy of both the lattice simulation and the SD equation.

\section{B. Features of gluodynamics in the asymptotic infrared limit}

We summarize the basic features of gluodynamics in the asymptotic infrared limit, under the assumption that the agreement between the SDE and the lattice gauge calculation is not accidental.

(i) The dynamics occurs in a single time slice. More precisely, the ghost and temporal gluon propagators, $D_{c \bar{c}}$ and $D_{A_{0} A_{0}}$ are both instantaneous, that is, proportional to $\delta\left(x_{0}-y_{0}\right)$, and the spatial gluon

${ }^{12}$ The numbers in the fourth column are close to the fractions $5 / 2,0,4$. which correspond to $d=3$. 
TABLE II. Comparison of Schwinger-Dyson equations to lattice.

\begin{tabular}{llllc}
\hline Critical exponents $\mathrm{LM}$ notation & SDE at $d_{c}$ & Lattice calculation \\
\hline$\alpha=(d+2) / 2=2 \kappa+2$ & $=2.4839$ & $\approx$ & $2.490(10)$ \\
$\gamma=0$ & $=0$ & $\approx$ & 0 \\
$\delta=d+1+\theta_{ \pm}(d)=2 \delta_{\mathrm{LM}}+2=3.9677$ & $\approx$ & $4.10(10)$ \\
\hline \hline
\end{tabular}

propagator is taken at equal time, $D_{\mathbf{A} \mathbf{A}}^{E T}(\mathbf{x}-\mathbf{y})=$ $D_{\mathbf{A A}}(t, \mathbf{x} ; t, \mathbf{y})$. This is due to the fact that in the Coulomb gauge, Gauss's law, $D_{i} \pi_{i}=\rho_{\text {quark }}$, is a constraint that is satisfied as an equation of motion.

(ii) In the asymptotic infrared limit, these propagators are fit by power laws with critical exponents whose values are given in the table.

(a) Compared to the tree-level propagator $1 /|\mathbf{k}|^{2}$, the ghost propagator is moderately long range.

(b) The color-Coulomb propagator is long-range, corresponding to a linear rise in $r$, or close to it.

(c) The infrared limit of the equal-time spatial gluon propagator has critical exponent 0 or close to $0 .{ }^{13}$

(iii) The horizon condition $\langle H(g A)\rangle=\left(N^{2}-1\right) d V$, and the divergence of the ghost dressing function, $\lim _{|\mathbf{k} \rightarrow 0|} \mathbf{k}^{2} D_{c \bar{c}}(\mathbf{k})=\infty$, are identical gauge conditions. This is shown in Apppendix A, and applied in Sec. VIII where the gauge condition is imposed by subtracting the $\mathbf{k}^{2}$ term in the SDE.

(iv) There is a shadow cast on these considerations because we have found no solution to the SDE at space dimension $d=3$, but only close to it, at $d=d_{c}=2.9677 \ldots$. We must figure out what mechanism, if any, acts so there is a solution at $d=3$. A small effect in the right direction would be sufficient. This could be provided by a dressed vertex replacing a tree-level vertex.

(v) In Appendix B, the contribution of gluon propagators to the Wilson loop $W=N^{-1} \operatorname{Tr} P \exp \left(\oint i g t^{b} A_{\mu}^{b} d x_{\mu}\right)$ is calculated. It is found that the spatial gluon propagator $D_{\mathbf{A}_{i} \mathbf{A}_{j}}$ does not contribute at all. Only the instantaneous temporal gluon propagator contributes, which moreover exponentiates, Eq. (B2). Consequently the calculation of the contribution of the gluon propagator to the path-ordered exponential is particularly simple in Coulomb gauge as compared to Lorentz-covariant gauges. This may be true for other expectation values.

\footnotetext{
${ }^{13}$ (a) and (b) are consistent with the Gribov confinement scenario. (c), on the other hand, is quite different. In the Gribov confinement scenario, the gluon propagator vanishes at $k=0$, which would automatically remove the gluon from the physical spectrum by having an unphysical spectral density. A nonvanishing, but constant propagator is, however, well supported in the lattice literature, despite the challenges of extracting infrared behavior on a finite lattice.
}

\section{ACKNOWLEDGMENTS}

D. Z. is grateful to Richard Brandt for many stimulating conversations.

\section{APPENDIX A: EQUIVALENCE OF HORIZON CONDITION AND THE MAXIMUM B-CONDITION}

The boundary of the (first) Gribov region $\partial \Omega$ is a set of transverse configurations $\partial_{i} A_{i}=0$ that satisfy the "horizon condition" [41],

$$
\langle H(g A)\rangle=\left(N^{2}-1\right) d V
$$

where $V=L^{d}$ is the spatial volume, the horizon function is defined by

$$
H(g A) \equiv \int d^{d} x d^{d} y D_{i \mathbf{x}}^{a b} D_{i \mathbf{y}}^{a c}\left(M^{-1}\right)_{\mathbf{x y}}^{b c}
$$

and $M^{a b}(g A)=-D_{i}^{a b}(g A) \partial_{i}$ is the Faddeev-Popov (FP) operator. The functional integral over the Gribov region $\Omega$ is equivalent to the functional integral over its boundary, ${ }^{14}$ the Gribov horizon $\partial \Omega$, and may be evaluated by insertion of $\delta\left[H-\left(N^{2}-1\right) d V\right][51]$.

We shall make use of two recent results [48]:

(1) The eigenvalues of the Faddeev-Popov operator $M^{a b}(g A)$ in the asymptotic infrared region $\mathbf{k} \rightarrow 0$ are given by

$$
\lambda_{|\mathbf{k}|}(g A)=\mathbf{k}^{2}\left(1-\frac{H(g A)}{\left(N^{2}-1\right) d V}+j_{|\mathbf{k}|}(g A)\right),
$$

where $\lambda_{|\mathbf{k}|}(g A)$ is the eigenvalue that emerges from $\lambda_{|\mathbf{k}|}(0)=\mathbf{k}^{2}$ at $A=0$, and $j_{|\mathbf{k}|}(g A)$ vanishes as $\mathbf{k}$ tends to 0 ,

$$
\lim _{\mathbf{k} \rightarrow 0} j_{|\mathbf{k}|}(g A)=0 .
$$

(2) In the asymptotic infrared region, the ghost propagator in an external gauge potential $\mathcal{G}(\mathbf{k} ; g A)=$ $\left\langle\vec{k}\left|\mathcal{M}^{-1}(g A)\right| \vec{k}\right\rangle$ is given by

$$
\mathcal{G}^{a b}(\mathbf{k}, g A)=\frac{\delta^{a b}}{\lambda_{|\mathbf{k}|}(g A)} .
$$

The horizon function is an instance of a bulk or extensive quantity in thermodynamics which, as explained in [41], Eq. (2.193), may be written as the integral of a density. Typically the mean and variance of a bulk quantity satisfy,

$$
\langle H(g A)\rangle=O(V) ; \quad H^{2}-\langle H\rangle^{2}=O(V) .
$$

\footnotetext{
${ }^{14}$ See footnote above.
} 
We assume that the horizon function behaves this way, and we easily find

$$
H(g A)=\langle H(g A)\rangle+O(1) .
$$

The fluctuations are down by order $1 / \mathrm{V}$ compared to the mean, $\langle H\rangle$. Thus, in the infinite-volume limit, $V \rightarrow \infty$, the horizon function $H(g A)$ may be replaced by its expectation-value $\langle H(g A)\rangle$, and the formula for the eigenvalues simplifies to

$$
\lambda_{|\mathbf{k}|}(g A)=\mathbf{k}^{2}\left(1-\frac{\langle H(g A)\rangle}{\left(N^{2}-1\right) d V}+j_{|\mathbf{k}|}(g A)\right) .
$$

We observe that when the horizon condition, Eq. (A1), is satisfied, the term of order $\mathbf{k}^{2}$ is precisely killed, so

$$
\lambda_{|\mathbf{k}|}(g A)=\mathbf{k}^{2} j_{|\mathbf{k}|}(g A),
$$

which gives

$$
\delta^{a b} b(\mathbf{k}) \equiv \delta^{a b} \mathbf{k}^{2} G(\mathbf{k})=\mathbf{k}^{2}\left\langle\mathcal{G}^{a b}(\mathbf{k}, g A)\right\rangle=\delta^{a b}\left\langle\frac{1}{j_{|\mathbf{k}|}(g A)}\right\rangle .
$$

Thus, if the horizon condition is satisfied, the ghost dressing function is divergent at $\mathbf{k}=0$,

$$
b(\mathbf{k}=0)=\infty .
$$

We call this the maximum- $b$ condition. The converse is also true: if the maximum-b condition is satisfied, then the horizon condition holds. We conclude that, in the $\Omega$-theory, the horizon condition and the maximum $b$-condition $b(0)=\infty$, are equivalent. Thus, it is justified to subtract the $\mathbf{k}^{2}$ term in the equation for the inverse ghost propagator $G^{-1}(\mathbf{k})$.

\section{APPENDIX B: CONTRIBUTION OF COULOMB GLUON PROPAGATORS TO WILSON LOOP}

We calculate the contribution of propagators to the Wilson loop, a gauge-invariant quantity.

Consider the expansion of the Wilson loop

$$
\begin{aligned}
W & =N^{-1} \operatorname{Tr} P \exp \left(i g \oint t^{a} A_{\mu}^{a} d x_{\mu}\right) \\
& =N^{-1} \sum_{n=0}^{\infty} \frac{1}{n !} \operatorname{Tr} P\left(i g \oint t^{a} A_{\mu}^{a} d x_{\mu}\right)^{n}
\end{aligned}
$$

in terms of connected and disconnected graphs. Let us set to zero all connected graphs that have three or more legs, so the Wilson loop is expressed as a sum of products of temporal and spatial gluon propagators $D_{\mu \nu}(x-y)$.
We shall show that under this simplifying assumption, (i) the spatial gluon propagators do not contribute to the Wilson loop, so only the color-Coulomb potential contributes to the forces on the Wilson loop, and (ii) the Wilson loop has the value

$$
W=\exp \left(-g^{2} C \int d x_{0} V\left[R\left(x_{0}\right)\right]\right),
$$

where $R\left(x_{0}\right)$ is the width of the Wilson loop as a function of Euclidean time. This situation is illustrated in Fig. 5, where all diagrams are ladder diagrams, and the (Euclidean) time direction is upward. This formula is the same as in an Abelian gauge theory, apart from the Casimir. Moreover, it implies that the contribution from the parallel parts of the Wilson loop (if any) is $W_{\text {parallel }}=\exp \left[-g^{2} C V(R) T\right]$, where the parallel parts are a distance $R$ apart for a time $T$.

Proof:-Recall that the temporal and spatial propagators vanish away from the time slice $x_{0}-y_{0}=0$,

$$
D_{\mu \nu}(x-y)=0 \quad \text { for } x_{0}-y_{0} \neq 0,
$$

but the temporal propagator $D_{A_{0} A_{0}}(x-y)=\delta\left(x_{0}-y_{0}\right) V(\mathbf{x}-\mathbf{y})$ has the factor $\delta\left(x_{0}-y_{0}\right)$, whereas the spatial propagator $D_{\mathbf{A}_{i} \mathbf{A}_{j}}(x-y)$ is finite at $x_{0}-y_{0}=0, D_{\mathbf{A}_{i} \mathbf{A}_{j}}(0, \mathbf{x}-\mathbf{y})=$ $D_{\mathbf{A}_{i} \mathbf{A}_{j}}^{E T}(\mathbf{x}-\mathbf{y})$. Consequently, when the line integral $\int d x_{\mu} D_{A_{\mu} A_{\nu}}\left(x_{0}-y_{0}, \mathbf{x}-\mathbf{y}\right)$ at fixed $y$, crosses the timeslice $x_{0}=y_{0}$, it receives a finite contribution if the gluon propagator is temporal $(\mu=0)$, but it receives no

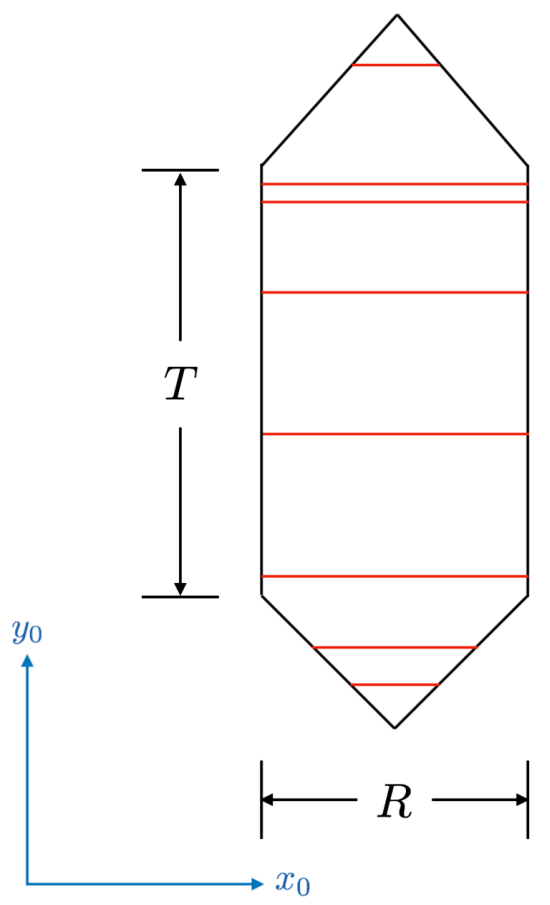

FIG. 5. Temporal gluon propagators form the rungs of Wilson loop, ladder diagrams. 
contribution when the gluon propagator is spatial $(\mu=1$, $2,3) .{ }^{15}$ Thus, the spatial gluon propagators do not contribute to the Wilson loop, as asserted. The remaining temporal propagators form the horizontal rungs of ladder diagrams, as illustrated in Fig. 5. The path-ordering makes the two ends of the lowest rung adjacent to each other so, for the lowest rung, the Lie algebra gives $t^{a} \delta^{a b} t^{b}=C_{F}$, where $C_{F}$ is the Casimir in the fundamental representation. Since it is proportional to the identity matrix, it may be removed from the path ordering. The same is then true for the next lowest rung etc., so path ordering gives a factor of $C_{F}^{n / 2}$. The combinatorics are then such that the propagators exponentiate exactly, ${ }^{16}$ and we obtain

$W=\exp \left[-\frac{1}{2} g^{2} C_{F} \oint d x_{\mu} \delta_{\mu 0} \oint d y_{\nu} \delta_{\nu 0} \delta\left(x_{0}-y_{0}\right) V(\mathbf{x}-\mathbf{y})\right]$

from which Eq. (B2) follows, as asserted. The important point is that the spatial gluon propagators have dropped out, and only the color-Coulomb potential contributes to the force on the Wilson loop. This is entirely consistent with numerical simulation [52] according to which the color-Coulomb electric field falls off exponentially. The last formula states that the color-Coulomb potential is the whole story. There is no contribution from the spatial gluon propagator.

In Lorentz-covariant gauges, such as the Landau gauge, the gluon propagator is not instantaneous, and whereas in Coulomb gauge the propagators form the horizontal rungs of a ladder and the path ordering is easily evaluated, as we have just seen, in a Lorentz-covariant gauge, the would-be rungs run every which way, and one does not know how to disentangle the path ordering. For this problem, calculation in the Coulomb gauge is simpler than in a Lorentzcovariant gauge.

\footnotetext{
${ }^{15}$ Here we suppose that the Wilson loop does not have a portion that lies within a time slice. This is the general case; the Wilson loop can be a circle or an ellipse or an upright diamond etc.

${ }^{16}$ The $n$th term in the power series expansion has $n !\left[2^{n / 2}(n / 2) !\right]^{-1}$ possible pairings when $n$ is even and vanishes when $n$ is odd.
}

\section{APPENDIX C: WIDTH OF THE NONINSTANTANEOUS GLUON PROPAGATOR VANISHES IN THE INFRARED LIMIT}

Suppose the critical exponents satisfy $\gamma=0$ and $\delta=$ $d+1$. Then by Eqs. (6.6) and (6.7) we have

$$
\begin{aligned}
\Gamma_{\mathbf{A A}} & =c_{1}|\mathbf{k}|^{d-\delta}=c_{1}|\mathbf{k}|^{-1} \\
\Gamma_{\tau \tau} & =c_{2}|\mathbf{k}|^{d-\delta}=c_{2}|\mathbf{k}|^{-1},
\end{aligned}
$$

where $c_{1}>0$ and $c_{2}>0$, which gives, by Eq. (6.4),

$$
\begin{aligned}
& \left(\begin{array}{cc}
D_{\tau \tau}\left(k_{0}, \mathbf{k}\right) & D_{\tau \mathbf{A}}\left(k_{0}, \mathbf{k}\right) \\
D_{\mathbf{A} \tau}\left(k_{0}, \mathbf{k}\right) & D_{\mathbf{A A}}\left(k_{0}, \mathbf{k}\right)
\end{array}\right) \\
& \quad=\frac{1}{k_{0}^{2}+c^{2} / \mathbf{k}^{2}}\left(\begin{array}{cc}
c_{1} /|\mathbf{k}| & k_{0} \\
-k_{0} & c_{2} /|\mathbf{k}|
\end{array}\right),
\end{aligned}
$$

where $c \equiv\left(c_{1} c_{2}\right)^{1 / 2}$. Upon taking the fourier transform, we obtain

$$
\begin{aligned}
& \left(\begin{array}{cc}
D_{\tau \tau}(t, \mathbf{k}) & D_{\tau \mathbf{A}}(t, \mathbf{k}) \\
D_{\mathbf{A} \tau}(t, \mathbf{k}) & D_{\mathbf{A A}}(t, \mathbf{k})
\end{array}\right) \\
& \quad=\frac{1}{2}\left(\begin{array}{cc}
\left(c_{1} / c_{2}\right)^{1 / 2} & \operatorname{sgn}(t) \\
-\operatorname{sgn}(t) & \left(c_{2} / c_{1}\right)^{1 / 2}
\end{array}\right) \exp (-c|t| /|\mathbf{k}|) .
\end{aligned}
$$

By comparison with $\langle E|\exp (-H t)| E\rangle=\langle E|\exp (-E t)| E\rangle$, we see that the state of a gluon of momentum $\mathbf{k}$ has energy

$$
E=c /|\mathbf{k}|
$$

Our power-law ansatz is valid only in the infrared limit $\mathbf{k} \rightarrow 0$. The transverse gluon propagator has a peak of width $w \sim|\mathbf{k}| / c$, which vanishes in the infrared limit, $w \rightarrow 0$, which corresponds to an instantaneous propagator at $\mathbf{k}=\mathbf{0}$. This resolves the apparent paradox mentioned in Sec. VI, that the time dependence of the propagators violate the remnant symmteries of Coulomb gauge. While our approximation scheme seems to break this symmetry, any inferences we make about the infrared dynamics will be free of this concern since the symmetry is restored at long distances.
[1] M. Teper, Acta Phys. Pol. B 40, 3249 (2009).

[2] C. S. Fischer and J. M. Pawlowski, Phys. Rev. D 75, 025012 (2007).

[3] T. Kugo and I. Ojima, Prog. Theor. Phys. Suppl. 66, 1 (1979).

[4] A. Weber, M. Leder, J. M. Pawlowski, and H. Reinhardt, J. Phys. Conf. Ser. 287, 012023 (2011).
[5] H. Reinhardt, M. Leder, J. M. Pawlowski, and A. Weber, AIP Conf. Proc. 1343, 164 (2011).

[6] C. S. Fischer and J. M. Pawlowski, Phys. Rev. D 80, 025023 (2009).

[7] A. L. Blum, R. Alkofer, M. Q. Huber, and A. Windisch, Acta Phys. Pol. B Proc. Suppl. 8, 321 (2015). 
[8] M. Vujinovic, R. Alkofer, G. Eichmann, and R. Williams, Acta Phys. Pol. B Proc. Suppl. 7, 607 (2014).

[9] P. Watson and H. Reinhardt, Proc. Sci., QCD-TNT-II2011 (2011) 051.

[10] P. Watson and H. Reinhardt, Phys. Rev. D 82, 125010 (2010).

[11] M. Q. Huber, R. Alkofer, and S. P. Sorella, AIP Conf. Proc. 1343, 158 (2011).

[12] M. Q. Huber, R. Alkofer, and S. P. Sorella, Phys. Rev. D 81, 065003 (2010).

[13] K. Lichtenegger and D. Zwanziger, arXiv:0911.5435.

[14] R. Alkofer, A. Maas, and D. Zwanziger, Few-Body Syst. 47, 73 (2010).

[15] D. R. Campagnari and H. Reinhardt, Phys. Rev. D 92, 065021 (2015).

[16] M. Q. Huber, D. R. Campagnari, and H. Reinhardt, Phys. Rev. D 91, 025014 (2015).

[17] H. Reinhardt and D. Campagnari, Proc. Sci., ConfinementX2012 (2012) 070.

[18] H. Reinhardt, D. R. Campagnari, and A. P. Szczepaniak, Phys. Rev. D 84, 045006 (2011).

[19] D. Epple, H. Reinhardt, and W. Schleifenbaum, Phys. Rev. D 75, 045011 (2007).

[20] W. Schleifenbaum, M. Leder, and H. Reinhardt, Phys. Rev. D 73, 125019 (2006).

[21] J. Greensite, S. Olejnik, and D. Zwanziger, Phys. Rev. D 69, 074506 (2004).

[22] F. Karsch and E. Laermann, arXiv:hep-lat/0305025.

[23] D. Zwanziger, Nucl. Phys. B518, 237 (1998).

[24] P. B. Arnold, Int. J. Mod. Phys. E 16, 2555 (2007).

[25] S. Dubovsky and V. Gorbenko, J. High Energy Phys. 02 (2016) 022.

[26] P. Cooper, S. Dubovsky, V. Gorbenko, A. Mohsen, and S. Storace, J. High Energy Phys. 04 (2015) 127.

[27] P. Cooper and D. Zwanziger, Phys. Rev. D 93, 105026 (2016).

[28] K. Langfeld and L. Moyaerts, Phys. Rev. D 70, 074507 (2004).
[29] G. Burgio, M. Quandt, and H. Reinhardt, Phys. Rev. D 86, 045029 (2012).

[30] Y. Nakagawa, A. Voigt, E. M. Ilgenfritz, M. MullerPreussker, A. Nakamura, T. Saito, A. Sternbeck, and H. Toki, Phys. Rev. D 79, 114504 (2009).

[31] A. Blum, M. Q. Huber, M. Mitter, and L. von Smekal, Phys. Rev. D 89, 061703 (2014).

[32] W. Schleifenbaum, A. Maas, J. Wambach, and R. Alkofer, Phys. Rev. D 72, 014017 (2005).

[33] A. Cucchieri, T. Mendes, and A. Mihara, J. High Energy Phys. 12 (2004) 012.

[34] J. C. Taylor, Nucl. Phys. B33, 436 (1971).

[35] J. C. R. Bloch, Few-Body Syst. 33, 111 (2003).

[36] R. N. Mohapatra, Phys. Rev. D 4, 1007 (1971).

[37] A. Andrasi and J. C. Taylor, Ann. Phys. (Amsterdam) 351, 407 (2014).

[38] A. Andrasi and J. C. Taylor, Eur. Phys. J. C 41, 377 (2005).

[39] A. Andrasi and J. C. Taylor, Ann. Phys. (Amsterdam) 356, 352 (2015).

[40] V. Gribov, Nucl. Phys. B139, 1 (1978).

[41] N. Vandersickel and D. Zwanziger, Phys. Rep. 520, 175 (2012).

[42] A. Maas, Phys. Lett. B 689, 107 (2010).

[43] A. Maas, Ann. Phys. (Amsterdam) 387, 29 (2017).

[44] D. Zwanziger, Nucl. Phys. B323, 513 (1989).

[45] D. Zwanziger, Nucl. Phys. B399, 477 (1993).

[46] Y. Nakagawa, A. Nakamura, T. Saito, and H. Toki, Phys. Rev. D 81, 054509 (2010).

[47] A. Sternbeck, E. M. Ilgenfritz, and M. Muller-Preussker, Phys. Rev. D 73, 014502 (2006).

[48] P. Cooper and D. Zwanziger, Phys. Rev. D 93, 105024 (2016).

[49] D. Zwanziger, Phys. Rev. D 69, 016002 (2004).

[50] D. Zwanziger, Phys. Rev. Lett. 90, 102001 (2003).

[51] D. Zwanziger, Phys. Rev. D 65, 094039 (2002).

[52] K. Chung and J. Greensite, Phys. Rev. D 96, 034512 (2017). 